\title{
Common fixed points of $\alpha$-dominated multivalued mappings on closed balls in a dislocated quasi b-metric space
}

\author{
Abdulaziz Saleem Moslem Alofia , Abdullah Eqal Al-Mazrooei ${ }^{\mathrm{a}, \mathrm{b}}$, Bahru Tsegaye Leyew ${ }^{\mathrm{c}}$, Mujahid Abbas $^{\mathrm{a}, \mathrm{d}, *}$ \\ a Department of Mathematics, King Abdulaziz University, P. O. Box 80203, Jeddah 21589, Saudi Arabia. \\ ${ }^{b}$ Department of Mathematics, University of Jeddah, P. O. Box 80327, Jeddah 21589, Saudi Arabia. \\ ${ }^{c}$ Department of Mathematics and Applied Mathematics, University of Pretoria, Lynnwood road, Pretoria 0002, South Africa. \\ ${ }^{d}$ Department of Mathematics, University of Management and Technology, C-Il Johar Town, Lahore, Pakistan.
}

Communicated by Z. Kadelburg

\begin{abstract}
In this paper, we introduce the concept of $\alpha$-dominated multivalued mappings and establish the existence of common fixed points of such mappings on a closed ball contained in left/right K-sequentially complete dislocated quasi b-metric spaces. These results improve, generalize, extend, unify, and complement various comparable results in the existing literature. Our results not only extend some primary results to left/right K-sequentially dislocated quasi b-metric spaces but also restrict the contractive conditions on a closed ball only. Some examples are presented to support the results proved herein. Finally as an application, we obtain some common fixed point results for single-valued mappings by an application of the corresponding results for multivalued mappings satisfying the contractive conditions more general than Banach type and Kannan type contractive conditions on closed balls in a left K-sequentially complete dislocated quasi b-metric space endowed with an arbitrary binary relation. (c) 2017 All rights reserved.
\end{abstract}

Keywords: K-sequentially complete, dislocated quasi b-metric spaces, $\alpha$-dominated multivalued mapping, closed ball, common fixed point.

2010 MSC: 47H04, 47H07, 47H10.

\section{Introduction and preliminaries}

The letters, $\mathbb{Q}, \mathbb{Q}^{+}, \mathbb{R}, \mathbb{R}^{+}, \mathbb{N}$, and $\mathbb{N}_{0}$ will denote the set of all rational numbers, the set of all nonnegative rational numbers, the set of all real numbers, the set of all nonnegative real numbers, and the set of all natural numbers and the set of all nonnegative integer numbers, respectively.

A point $x$ in a nonempty set $X$ is called a fixed point of the mapping $T: X \rightarrow X$ if $T x=x$. In metric fixed point theory, imposition of a contractive condition on a mapping plays an important role for proving the existence of a fixed point of a mapping (see, $[6,7,14,17,23]$ and references therein).

Let $(X, d)$ be a metric space. A mapping $T: X \rightarrow X$ is called:

\footnotetext{
*Corresponding author

Email addresses: aalofi1@kau.edu.sa (Abdulaziz Saleem Moslem Alofi), aealmazrooei@uj .edu.sa (Abdullah Eqal Al-Mazrooei), tsegayebah@gmail.com (Bahru Tsegaye Leyew), mujahid.abbas@up.ac.za (Mujahid Abbas) 
(BC) Banach contraction mapping [7] if for any $x, y \in X$, there exists $k \in[0,1)$ such that

$$
d(T x, T y) \leqslant k d(x, y)
$$

(KN) Kannan contraction mapping [17] if there exists $k \in\left[0, \frac{1}{2}\right)$ such that

$$
d(T x, T y) \leqslant k(d(x, T x)+d(y, T y))
$$

holds for all $x, y \in X$.

If either of the conditions $(\mathrm{BC})$ or $(\mathrm{KN})$ is fulfilled, then $\mathrm{T}$ has a unique fixed point provided that $\mathrm{X}$ is a complete metric space. The mapping $T$ satisfying the condition $(\mathrm{BC})$ is continuous while the condition (KN) does not ensure the continuity of a mapping T. This makes Kannan contraction mappings more important than the mappings satisfying Banach contraction condition (BC).

On the other hand, the concept of a metric space has been generalized in several ways.

Definition 1.1 ([35]). Let $X$ be a nonempty set and $d: X \times X \rightarrow \mathbb{R}^{+}$. Suppose that for any $x, y, z \in X$, the following conditions hold:

$\left(\mathrm{d}_{1}\right) \mathrm{d}(\mathrm{x}, \mathrm{x})=0$;

$\left(d_{2}\right) d(x, y)=d(y, x)=0$ implies that $x=y$;

$\left(d_{3}\right) d(x, y)=d(y, x)$;

$\left(d_{4}\right) d(x, y) \leqslant d(x, z)+d(z, y)$.

If $d$ satisfies conditions $\left(d_{2}\right)$ to $\left(d_{4}\right)$, then $d$ is called a dislocated metric on $X$. If $d$ satisfies conditions $\left(d_{1}\right)$, $\left(d_{2}\right)$, and $\left(d_{4}\right)$, then $d$ is called a quasi metric on $X$. If $d$ satisfies conditions $\left(d_{2}\right)$ and $\left(d_{4}\right)$, then $d$ is called a dislocated quasi metric (dq metric) on $X$. If $d$ satisfies conditions $\left(d_{1}\right)$ to $\left(d_{4}\right)$, then $d$ is called a metric on X.

In 1931, Wilson [34] introduced the notion of quasi metric space as a generalization of metric space. In 2000, Hitzler et al. [13] introduced dislocated metric spaces (metric like spaces). The notion of dislocated topologies have useful applications in the context of logic programming semantics $([12,13])$. Combining the concepts of quasi and dislocated metric spaces, Zeyada et al. [35] coined the notion of a dislocated quasi metric space (quasi-metric like space).

Definition $1.2([2,6,18,32])$. Let $X$ be a nonempty set and $s \geqslant 1$ a real number. Suppose that for any $x, y, z \in X$, the mapping $d_{q b}: X \times X \rightarrow \mathbb{R}^{+}$satisfies the following conditions:

$\left(\mathrm{dq}_{1}\right) \mathrm{d}_{\mathrm{qb}}(\mathrm{x}, \mathrm{x})=0$;

$\left(d_{2}\right) d_{q b}(x, y)=d_{q b}(y, x)=0$ implies $x=y$;

$\left(d_{1}\right) d_{q b}(x, y)=d_{q b}(y, x)$;

$\left(d_{4}\right) d_{q b}(x, y) \leqslant s\left(d_{q b}(x, z)+d_{q b}(z, y)\right)$.

If $\mathrm{d}_{\mathrm{qb}}$ satisfies conditions $\left(\mathrm{dq}_{1}\right),\left(\mathrm{dq}_{2}\right)$, and $\left(\mathrm{dq}_{4}\right)$, then $\mathrm{d}_{\mathrm{qb}}$ is called a quasi b-metric on $X$ [32]. If $d_{q b}$ satisfies conditions $\left(d_{2}\right)$ to $\left(d_{q_{4}}\right)$, then $d_{q b}$ is called a dislocated b-metric on $X$ [2]. If $d_{q b}$ satisfies conditions $\left(\mathrm{dq}_{2}\right)$ and $\left(\mathrm{dq}_{4}\right)$, then $\mathrm{d}_{\mathrm{qb}}$ is called a dislocated quasi b-metric (or simply $\mathrm{dq} \mathrm{b}$-metric) on $\mathrm{X}$ [18]. If $d_{q b}$ satisfies conditions $\left(d_{q_{1}}\right)$ to $\left(d_{q_{4}}\right)$, then $d_{q b}$ is called a b-metric on $X$ [6].

Czerwik [10] proved Banach contraction theorem for single and multivalued mappings in b-metric spaces. Since then several fixed point results for various classes of single-valued and multivalued operators have been proved in the framework of b-metric spaces [10, 11, 29]. The concepts of quasi b-metric, dislocated b-metric, and dq b-metric are more general than that of a b-metric.

Remark 1.3. In Definition 1.2, if $s=1$, then

1. b-metric space is a metric space; 
2. quasi b-metric space is a quasi metric space;

3. dislocated b-metric space (or b-metric-like space) is a dislocated metric space (or metric-like space);

4. dq b-metric space (or quasi b-metric-like space) is a dq metric space (or quasi metric-like space).

Note that a b-metric $d_{q b}: X \times X \rightarrow \mathbb{R}^{+}$is not necessarily continuous in each variable. Also, if b-metric $\mathrm{d}_{\mathrm{qb}}$ is continuous in one variable, then it is continuous in the other variable (see [3]).

It is obvious that b-metric spaces, quasi b-metric spaces, and dislocated b-metric spaces are dq $b$-metric spaces, but the converse does not hold in general.

Example 1.4 ([18, Example 2.3]). Let $X=\mathbb{R}$. Define $d_{q b}: X \times X \rightarrow \mathbb{R}^{+}$by

$$
d_{q b}(x, y)=|x-y|^{2}+\frac{|x|}{n}+\frac{|y|}{m}
$$

where $m, n \in \mathbb{N} \backslash\{1\}$ with $n \neq m$. Then $\left(X, d_{q b}\right)$ is a dq b-metric space with $s=2$. As $d_{q b}(1,1) \neq 0$, $\left(X, d_{q b}\right)$ is not a quasi b-metric space. Note that $d_{q b}(1,2) \neq d_{q b}(2,1)$. Thus $\left(X, d_{q b}\right)$ is not a dislocated $b$-metric space. Also, $\left(X, d_{q b}\right)$ is not a dislocated quasi metric space.

Example 1.5 ([36, Example2.1]). Let $X=\{0,1,2\}$. Define $d_{\mathrm{qb}}: X \times X \rightarrow \mathbb{R}^{+}$by

$$
d_{\mathrm{qb}}(x, y)= \begin{cases}2, & x=y=0 \\ \frac{1}{2}, & x=0, y=1 \\ 2, & x=1, y=0 \\ \frac{1}{2}, & \text { otherwise }\end{cases}
$$

Then $\left(X, d_{q b}\right)$ is a dq b-metric space with $s=2$. As $d_{q b}(1,1) \neq 0,\left(X, d_{q b}\right)$ is not a quasi b-metric space. Also $\mathrm{d}_{\mathrm{qb}}(0,1) \neq \mathrm{d}_{\mathrm{qb}}(1,0)$ implies that $\left(X, \mathrm{~d}_{\mathrm{qb}}\right)$ is not a dislocated $b$-metric space. It is obvious that $\left(X, d_{q b}\right)$ is not a dislocated quasi metric space.

In view of the following proposition, some more examples of dq b-metric spaces can easily be constructed.

Proposition 1.6 ([26]). Let $\mathrm{X}$ be a nonempty set such that $\mathrm{d}_{\mathrm{q}}$ is a dq metric and $\mathrm{d}_{\mathrm{b}}$ is a b-metric with $\mathrm{s}>1$ on $\mathrm{X}$. Then the function $\mathrm{d}_{\mathrm{qb}}: \mathrm{X} \times \mathrm{X} \rightarrow \mathbb{R}^{+}$defined by

$$
d_{q b}(x, y)=d_{q}(x, y)+d_{b}(x, y)
$$

is $d q$-metric on $\mathrm{X}$.

Reilly et al. [28] introduced the concept of left/right K-Cauchy sequence and left/right K-sequentially complete spaces (see [5]). We have the following definitions given in [33, 36].

Definition 1.7. Let $\left(X, d_{q b}\right)$ be a dq b-metric space. A sequence $\left\{x_{n}\right\}$ in $\left(X, d_{q b}\right)$ is called:

(a) $d q$ b-converges to a point $x \in X$ if and only if

$$
\lim _{n \rightarrow \infty} d_{q b}\left(x_{n}, x\right)=0=\lim _{n \rightarrow \infty} d_{q b}\left(x, x_{n}\right),
$$

in this case $x$ is called a dq b-limit of $\left\{x_{n}\right\}$ and we write $x_{n} \rightarrow x$ as $n \rightarrow \infty$;

(b) left (right) K-Cauchy sequence if $\forall \epsilon>0$, there exists $n_{0} \in \mathbb{N}$ such that for all $n>m \geqslant n_{0}$, $d_{q b}\left(x_{m}, x_{n}\right)<\epsilon\left(d_{q b}\left(x_{n}, x_{m}\right)<\epsilon\right)$.

The space $\left(X, d_{q b}\right)$ is called left (right) K-sequentially complete if every left (right) K-Cauchy sequence in $\mathrm{X} \mathrm{dq} \mathrm{b}$-converges to a point $\mathrm{x} \in \mathrm{X}$.

Each dq b-metric $d_{q b}$ generates a topology on $X$ whose base is the family of open balls $\left\{B_{q b}\left(x_{0}, r\right)\right.$ : $\left.x_{0} \in X, r>0\right\}$, where $B_{q b}\left(x_{0}, r\right)=\left\{x \in X: \max \left\{d_{q b}\left(x_{0}, x\right), d_{q b}\left(x, x_{0}\right)\right\}<r\right\}$. The closure of $B_{q b}\left(x_{0}, r\right)$ is denoted by $B_{q b}\left[x_{0}, r\right]$. 
Throughout this paper, we assume that a $d q b$-metric $d_{q b}$ is continuous in one variable.

The development of metric fixed point theory for multivalued mappings was initiated by Nadler [23] in 1969. He introduced the concept of set-valued contraction mappings and extended the Banach contraction principle to set-valued mappings by using the Hausdorff metric. Since then various wellknown results for single-valued contraction mappings have been extended for multivalued mappings (see, [9-11, 16, 19-21, 32] and references mentioned therein).

Arshad et al. [4] proved some results dealing with the fixed points of a mapping satisfying a contractive conditions on closed ball contained in a complete dislocated metric space. For more results in this direction, we refer to $[4,5]$ and references mentioned therein. These results are very useful in the sense that they require the contraction condition of the mapping only on the closed ball instead of the entire space.

Consistent with $[2,3,6,8,10,11,15,18,30,32]$, the following definitions and results will be needed to derive the main results.

Unless stated otherwise from now onwards, $X$ denotes $d q b$-metric space equipped with dq $b$-metric $\mathrm{d}_{\mathrm{qb}}$ with constant $\mathrm{s} \geqslant 1$. Suppose that

$$
\begin{aligned}
P(X) & =\{A: A \text { is a subset of } X\} \\
N(X) & =\{A: A \text { is a nonempty subset of } X\} \\
B(X) & =\{A: A \text { is a nonempty bounded subset of } X\} \\
C L(X) & =\{A: A \text { is a nonempty closed subset of } X\} \\
C(X) & =\{A: A \text { is a nonempty compact subset of } X\}, \\
C B(X) & =\{A: A \text { is a nonempty closed and bounded subset of } X\} .
\end{aligned}
$$

Let $S, T: X \rightarrow N(X)$. A point $x^{*} \in X$ is called:

(1) a fixed point of $\mathrm{T}$ if $x^{*} \in \mathrm{T} x^{*}$;

(2) a common fixed point of $S$ and $T$ if $x^{*} \in S x^{*} \cap T x^{*}$.

We denote by $F(T)$ the set of fixed point of $T$.

For $A, B \in C B(X)$ and $x \in X$, define

and

$$
\begin{aligned}
& \delta_{q b}(A, B)=\sup \left\{d_{q b}(x, B): x \in A\right\}, \\
& \delta_{q b}(B, A)=\sup \left\{d_{q b}(y, A): y \in B\right\},
\end{aligned}
$$

$$
H_{q b}(A, B)=\max \left\{\delta_{q b}(A, B), \delta_{q b}(B, A)\right\}
$$

where

$$
d_{q b}(x, B)=\inf \left\{d_{q b}(x, y): y \in B\right\} .
$$

The function $\mathrm{H}_{\mathrm{qb}}$ is called the Hausdorff $\mathrm{dq} b$-metric on $\mathrm{CB}(X)$ induced by $\mathrm{d}_{\mathrm{qb}}$. Note that, $\mathrm{H}_{\mathrm{qb}}(A, B) \leqslant$ $s\left(H_{q b}(A, C)+H_{q b}(C, B)\right)$. Also, $H_{q b}(A, B)=0$ implies that $A=B$. Furthermore, $\left(C B(X), H_{q b}\right)$ is complete if $\left(X, d_{q b}\right)$ is complete.

A mapping $T: X \rightarrow C L(X)$ is said to be continuous at $x \in X$ if for every sequence $\left\{x_{n}\right\}$ in $X$ with $\lim _{n \rightarrow \infty} d_{d q}\left(x_{n}, x\right)=0$, we have

$$
\lim _{n \rightarrow \infty} H_{d q}\left(T x_{n}, T x\right)=0 .
$$

Definition 1.8. Let $X$ be a nonempty set and $\alpha: X \times X \rightarrow \mathbb{R}^{+}$. A mapping $T: X \rightarrow N(X)$ is called $\alpha$-dominated on $X$ if for each $x \in X$, we have $\alpha(x, u) \geqslant 1$ for any $u \in T x$.

$T$ is $\alpha$-dominated on $A \subseteq X$ if for each $x \in A$, we have $\alpha(x, u) \geqslant 1$ for any $u \in T x$. 
Example 1.9. Let $X=\{0,1,2\}$. Define the mapping $T: X \rightarrow N(X)$ by

$$
T x= \begin{cases}\left\{\frac{x}{2}, \frac{x+2}{2}\right\}, & \text { if } x \in\{0,2\}, \\ \{x-1, x\}, & \text { otherwise, }\end{cases}
$$

and $\alpha: X \times X \rightarrow \mathbb{R}^{+}$as:

$$
\alpha(x, y)= \begin{cases}e^{x+y}, & \text { if } x=y, \\ 1, & \text { otherwise. }\end{cases}
$$

Note that, $\mathrm{T} 0=\{0,1\}, \mathrm{T} 1=\{0,1\}$, and $\mathrm{T} 2=\{1,2\}$. Note that for each $x \in X, \alpha(x, u) \geqslant 1$ for any $u \in T x$ and hence $T$ is $\alpha$-dominated mapping on $X$.

Example 1.10. Let $X=\mathbb{R}^{+}$. Define $T: X \rightarrow N(X)$ by

$$
T x= \begin{cases}{\left[0, \frac{x}{4}\right],} & \text { if } x \in\left[0, \frac{1}{2}\right], \\ {\left[\frac{x}{2}, x\right],} & \text { if } x \in\left(\frac{1}{2}, 1\right], \\ \{x\}, & \text { otherwise, }\end{cases}
$$

and $\alpha: X \times X \rightarrow \mathbb{R}^{+}$as:

$$
\alpha(x, y)= \begin{cases}\cosh (x+y), & \text { if } x, y \in[0,1] \\ \tanh \left(x^{2}+y^{2}\right), & \text { otherwise }\end{cases}
$$

Note that for any $x \in X$, the set $T x$ is closed subset of $X$. Clearly, $T$ is $\alpha$-dominated mapping on $[0,1]$. Indeed,

(1) if $x \in\left[0, \frac{1}{2}\right]$, then $T x=\left[0, \frac{x}{4}\right] \subseteq\left[0, \frac{1}{8}\right]$. Hence $\alpha(x, y)=\cosh (x+y) \geqslant 1$ for all $y \in T x$;

(2) if $x \in\left(\frac{1}{2}, 1\right]$, then $T x=\left[\frac{x}{2}, x\right] \subseteq\left(\frac{1}{4}, 1\right]$. Hence $\alpha(x, y)=\cosh (x+y) \geqslant 1$ for all $y \in T x$;

(3) if $x \in(1, \infty)$, then $T x=\{x\} \subseteq(1, \infty)$. Hence $\alpha(x, y)=\tanh \left(x^{2}+y^{2}\right)<1$ for all $y \in T x$.

We need the following analogous Lemmas [23] in the framework of $d_{q b}$ metric spaces. For sake of completeness, we give the proofs.

Lemma 1.11. Let $A, B \in C B(X)$. If $a \in A$, then $d_{q b}(a, B) \leqslant H_{q b}(A, B)$.

Proof. $d_{q b}(a, B) \leqslant \sup \left\{d_{q b}(x, B): x \in A\right\}=\delta_{q b}(A, B) \leqslant H_{q b}(A, B)$.

Lemma 1.12. If $A, B \in C B(X)$ and $\varepsilon>0$, then for $a \in A$ there exists $b \in B$ such that $d_{q b}(a, b) \leqslant H_{q b}(A, B)+\varepsilon$.

Proof. Assume on contrary that there exists $\varepsilon>0$ such that for any $b \in B$, we have

$$
d_{q b}(a, b)>H_{q b}(A, B)+\varepsilon .
$$

Then,

$$
d_{q b}(a, B)=\inf \left\{d_{q b}(a, b): b \in B\right\} \geqslant H_{q b}(A, B)+\varepsilon \geqslant \delta_{q b}(A, B)+\varepsilon=\sup \left\{d_{q b}(x, B): x \in A\right\}+\varepsilon,
$$

a contradiction.

Lemma 1.13. Let $A, B \in C B(X)$ and $\mu>1$. Then for every $a \in A$, there exists $b \in B$ such that $d(a, b) \leqslant$ $\mu \mathrm{H}_{\mathrm{qb}}(\mathrm{A}, \mathrm{B})$.

Proof. Suppose that $A=B$ and $a \in A$. Then, we have

$$
\mathrm{H}_{\mathrm{qb}}(A, B)=\mathrm{H}_{\mathrm{qb}}(A, A)=\delta_{\mathrm{qb}}(A, A)=\sup \left\{\mathrm{d}_{\mathrm{qb}}(x, x): x \in A\right\},
$$

and hence

$$
d_{q b}(a, a) \leqslant \sup \left\{d_{q b}(x, x): x \in A\right\}=H_{q b}(A, B) \leqslant \mu H_{q b}(A, B) .
$$


Thus $b=a$ satisfies $d_{q b}(a, b) \leqslant \mu H_{q b}(A, B)$. Let $A \neq B$. Assume that there exists $a \in A$ such that $d_{q b}(a, b)>\mu H_{q b}(A, B)$ for all $b \in B$. Then,

$$
\mathrm{d}_{\mathrm{qb}}(\mathrm{a}, \mathrm{B})=\inf \left\{\mathrm{d}_{\mathrm{qb}}(\mathrm{a}, z): z \in \mathrm{B}\right\} \geqslant \mu \mathrm{H}_{\mathrm{qb}}(\mathrm{A}, \mathrm{B}) .
$$

Note that

$$
\mathrm{H}_{\mathrm{qb}}(A, B) \geqslant \delta_{\mathrm{qb}}(A, B)=\sup \left\{\mathrm{d}_{\mathrm{qb}}(x, B): x \in A\right\} \geqslant \mathrm{d}_{\mathrm{qb}}(\mathrm{a}, \mathrm{B}) \geqslant \mu \mathrm{H}_{\mathrm{qb}}(\mathrm{A}, \mathrm{B}) .
$$

As $H_{q b}$ is dislocated and $A \neq B, H_{q b}(A, B) \neq 0$. Thus $\mu \leqslant 1$, a contradiction.

Lemma 1.14. Let $A, B \in C(X)$ and $\mu \geqslant 1$. Then for every $a \in A$, there exists $b \in B$ such that $d(a, b) \leqslant$ $\mu \mathrm{H}_{\mathrm{qb}}(\mathrm{A}, \mathrm{B})$.

From the viewpoint of application of contraction mapping, it is possible that a mapping $T$ defined on the space $X$ satisfies contractive condition on the subset $Y$ of the space $X$ rather than on the entire space $X$. In addition, the contraction mapping under consideration may not be continuous. In this paper, we establish the existence of common fixed points for multivalued $\alpha$-dominated mappings which are assumed to satisfy contractive conditions only on a closed ball in dq b-metric spaces. We also obtain certain fixed point and common fixed point theorems of multivalued mappings on a complete dq bmetric space endowed with an arbitrary binary relation. We apply our results to prove the existence of fixed point and common fixed point of single-valued mappings in the setup of dislocated quasi b-metric spaces.

\section{Main results}

In this section, we present some results dealing with the existence of common fixed point for $\alpha$ dominated multivalued mappings satisfying certain contractive conditions on closed balls in the framework of left K-sequentially complete dq b-metric spaces.

We start with the following result.

Theorem 2.1. Let $\left(\mathrm{X}, \mathrm{d}_{\mathrm{qb}}\right)$ be a left $\mathrm{K}$-sequentially complete $d q$ b-metric space, $\mathrm{x}_{0} \in \mathrm{X}$, and $\alpha: \mathrm{X} \times \mathrm{X} \rightarrow \mathbb{R}^{+}$. Suppose that $\mathrm{S}, \mathrm{T}: \mathrm{X} \rightarrow \mathrm{CB}(\mathrm{X})$ are $\alpha$-dominated mappings on $\mathrm{B}_{\mathrm{qb}}\left[\mathrm{x}_{0}, \mathrm{r}\right]$. If

(i) for any $x, y \in B_{q b}\left[x_{0}, r\right]$ with $\alpha(x, y) \geqslant 1$, we have

$$
H_{q b}(S x, T y) \leqslant \lambda d_{q b}(x, y)+\beta\left[d_{q b}(x, S x)+d_{q b}(y, T y)\right],
$$

and

$$
H_{q b}(T x, S y) \leqslant \lambda d_{q b}(x, y)+\beta\left[d_{q b}(x, T x)+d_{q b}(y, S y)\right],
$$

where $0 \leqslant \lambda+2 \beta<1$;

(ii) there exists $\mathrm{x}_{1} \in \mathrm{S} \mathrm{x}_{0}$ such that

$$
d_{q b}\left(x_{0}, x_{1}\right) \leqslant k(1-s k) r
$$

(iii) either

holds, where $\mathrm{k}=\frac{\lambda+\beta}{1-\beta}$ and $\mathrm{sk}<1$;

(a) S, T are continuous; or

(b) for any sequence $\left\{x_{n}\right\}$ in $B_{\mathrm{qb}}\left[x_{0}, r\right]$ with $\alpha\left(x_{n}, x_{n+1}\right) \geqslant 1$ for all $n \in \mathbb{N}_{0}$ and $x_{n} \rightarrow x \in B_{q b}\left[x_{0}, r\right]$ as $\mathrm{n} \rightarrow \infty$, we have $\alpha\left(\mathrm{x}_{\mathrm{n}}, \mathrm{x}\right) \geqslant 1$ for all $\mathrm{n} \in \mathbb{N}_{0}$,

then $\mathrm{S}$ and $\mathrm{T}$ have a common fixed point $\mathrm{x}^{*} \in \mathrm{B}_{\mathrm{qb}}\left[\mathrm{x}_{0}, \mathrm{r}\right]$. If, in addition, $\mathrm{S}$ and $\mathrm{T}$ are compact valued mappings, then $\mathrm{d}_{\mathrm{qb}}\left(\mathrm{x}^{*}, \mathrm{x}^{*}\right)=0$.

Proof. Let $\mathrm{x}_{0}$ be a given point in $\mathrm{X}$ and $\mathrm{x}_{1} \in \mathrm{S} \mathrm{x}_{0}$. Note that,

$$
d_{q b}\left(x_{0}, x_{1}\right) \leqslant k(1-s k) r \leqslant r .
$$

Hence $x_{1} \in B_{\mathrm{qb}}\left[x_{0}, r\right]$. Choose $\varepsilon>0$ such that 


$$
k d_{\mathrm{qb}}\left(\mathrm{x}_{0}, \mathrm{x}_{1}\right)+\frac{\varepsilon}{1-\beta} \leqslant \mathrm{k}^{2}(1-\mathrm{sk}) \mathrm{r}
$$

holds. Since $x_{1} \in S x_{0}$, and $S$ is $\alpha$-dominated mapping on $B_{q b}\left[x_{0}, r\right]$, we have $\alpha\left(x_{0}, x_{1}\right) \geqslant 1$. By Lemma 1.12 , there exists $x_{2} \in T x_{1}$ such that

$$
\mathrm{d}_{\mathrm{qb}}\left(\mathrm{x}_{1}, \mathrm{x}_{2}\right) \leqslant \mathrm{H}_{\mathrm{qb}}\left(S \mathrm{x}_{0}, \mathrm{~T} \mathrm{x}_{1}\right)+\varepsilon .
$$

It follows from (2.1) that

$$
\begin{aligned}
d_{q b}\left(x_{1}, x_{2}\right) & \leqslant \lambda d_{q b}\left(x_{0}, x_{1}\right)+\beta\left[d_{q b}\left(x_{0}, S x_{0}\right)+d_{q b}\left(x_{1}, T x_{1}\right)\right]+\varepsilon \\
& \leqslant \lambda d_{q b}\left(x_{0}, x_{1}\right)+\beta\left[d_{q b}\left(x_{0}, x_{1}\right)+d_{q b}\left(x_{1}, x_{2}\right)\right]+\varepsilon \\
& \leqslant(\lambda+\beta) d_{q b}\left(x_{0}, x_{1}\right)+\beta d_{q b}\left(x_{1}, x_{2}\right)+\varepsilon,
\end{aligned}
$$

that is,

which further implies that

$$
d_{q b}\left(x_{1}, x_{2}\right) \leqslant k d_{q b}\left(x_{0}, x_{1}\right)+\frac{\varepsilon}{1-\beta}
$$

$$
d_{q b}\left(x_{1}, x_{2}\right) \leqslant k^{2}(1-s k) r
$$

Thus from (2.3) and (2.4), we have

$$
\begin{aligned}
\mathrm{d}_{\mathrm{qb}}\left(x_{0}, x_{2}\right) & \leqslant s\left\{\mathrm{~d}_{\mathrm{qb}}\left(x_{0}, x_{1}\right)+\mathrm{d}_{\mathrm{qb}}\left(x_{1}, x_{2}\right)\right\} \\
& \leqslant s k(1-s k) r+s k^{2}(1-s k) r \\
& \leqslant s k(1-s k) r+(s k)^{2}(1-s k) r \\
& \leqslant s k(1-s k) r[1+s k] \\
& \leqslant s k(1-s k) r\left[1+s k+(s k)^{2}+\cdots\right] \\
& =(s k)(1-s k) r \frac{1}{1-s k} \leqslant r
\end{aligned}
$$

which shows that $x_{2} \in B_{\mathrm{qb}}\left[x_{0}, r\right]$. Choose $\varepsilon>0$ such that

$$
k^{2} d_{q b}\left(x_{0}, x_{1}\right)+\frac{\varepsilon}{1-\beta}(1+k) \leqslant k^{3}(1-s k) r
$$

holds. By Lemma 1.12, there exists $x_{3} \in S x_{2}$ such that

$$
\mathrm{d}_{\mathrm{qb}}\left(\mathrm{x}_{2}, \mathrm{x}_{3}\right) \leqslant \mathrm{H}_{\mathrm{qb}}\left(T x_{1}, S x_{2}\right)+\varepsilon .
$$

Since $x_{2} \in T x_{1}$, and $T$ is $\alpha$-dominated mapping, we get $\alpha\left(x_{1}, x_{2}\right) \geqslant 1$. It follows from (2.2) that

$$
\begin{aligned}
d_{q b}\left(x_{2}, x_{3}\right) & \leqslant \lambda d_{q b}\left(x_{1}, x_{2}\right)+\beta\left[d_{q b}\left(x_{1}, T x_{1}\right)+d_{q b}\left(x_{2}, S x_{2}\right)\right]+\varepsilon \\
& \leqslant \lambda d_{q b}\left(x_{1}, x_{2}\right)+\beta\left[d_{q b}\left(x_{1}, x_{2}\right)+d_{q b}\left(x_{2}, x_{3}\right)\right]+\varepsilon \\
& \leqslant(\lambda+\beta) d_{q b}\left(x_{1}, x_{2}\right)+\beta d_{q b}\left(x_{2}, x_{3}\right)+\varepsilon,
\end{aligned}
$$

that is,

$$
\mathrm{d}_{\mathrm{qb}}\left(\mathrm{x}_{2}, \mathrm{x}_{3}\right) \leqslant k \mathrm{~d}_{\mathrm{qb}}\left(\mathrm{x}_{1}, \mathrm{x}_{2}\right)+\frac{\varepsilon}{1-\beta} \leqslant \mathrm{k}\left(\mathrm{kd}_{\mathrm{qb}}\left(\mathrm{x}_{0}, \mathrm{x}_{1}\right)+\frac{\varepsilon}{1-\beta}\right)+\frac{\varepsilon}{1-\beta} \leqslant \mathrm{k}^{2} \mathrm{~d}_{\mathrm{qb}}\left(\mathrm{x}_{0}, \mathrm{x}_{1}\right)+\frac{\varepsilon}{1-\beta}(1+\mathrm{k}),
$$

which further implies that

$$
d_{q b}\left(x_{2}, x_{3}\right) \leqslant k^{3}(1-s k) r .
$$

Thus from (2.3), (2.4), and (2.5), we have

$$
d_{q b}\left(x_{0}, x_{3}\right) \leqslant s\left\{d_{q b}\left(x_{0}, x_{1}\right)+d_{q b}\left(x_{1}, x_{3}\right)\right\}
$$




$$
\begin{aligned}
& \leqslant s\left\{d_{q b}\left(x_{0}, x_{1}\right)+s\left(d_{q b}\left(x_{1}, x_{2}\right)+d_{q b}\left(x_{2}, x_{3}\right)\right)\right\} \\
& \leqslant s k(1-s k) r+s^{2} k^{2}(1-s k) r+s^{2} k^{3}(1-s k) r \\
& \leqslant s k(1-s k) r+(s k)^{2}(1-s k) r+s^{3} k^{3}(1-s k) r \\
& \leqslant s k(1-s k) r\left[1+s k+(s k)^{2}\right] \\
& \leqslant s k(1-s k) r\left[1+s k+(s k)^{2}+\cdots\right] \\
& =(s k)(1-s k) r \frac{1}{1-s k} \leqslant r,
\end{aligned}
$$

which shows that $x_{3} \in B_{q b}\left[x_{0}, r\right]$. Let $x_{2 i} \in B_{q b}\left[x_{0}, r\right]$ for some $i \in \mathbb{N}$ with $x_{2 i} \in T x_{2 i-1}$ and

$$
d_{q b}\left(x_{2 i-1}, x_{2 i}\right) \leqslant k^{2 i-1} d_{q b}\left(x_{0}, x_{1}\right)+\frac{\varepsilon}{1-\beta} \sum_{p=0}^{2 i-2} k^{p} \leqslant k^{2 i}(1-s k) r .
$$

Now choose $\varepsilon>0$ such that

$$
k^{2 i} d_{q b}\left(x_{0}, x_{1}\right)+\frac{\varepsilon}{1-\beta} \sum_{p=0}^{2 i-1} k^{p} \leqslant k^{2 i+1}(1-s k) r .
$$

Since $x_{2 i} \in T x_{2 i-1}$ and $T$ is $\alpha$-dominated mapping on $B_{q b}\left[x_{0}, r\right]$, we have $\alpha\left(x_{2 i-1}, x_{2 i}\right) \geqslant 1$. By Lemma 1.12 , there exists $x_{2 i+1} \in S x_{2 i}$ such that

$$
\mathrm{d}_{\mathrm{qb}}\left(\mathrm{x}_{2 i}, \mathrm{x}_{2 i+1}\right) \leqslant \mathrm{H}_{\mathrm{qb}}\left(T x_{2 i-1}, S x_{2 i}\right)+\varepsilon .
$$

It follows from (2.2) that

$$
\begin{aligned}
d_{q b}\left(x_{2 i}, x_{2 i+1}\right) & \leqslant \lambda d_{q b}\left(x_{2 i-1}, x_{2 i}\right)+\beta\left[d_{q b}\left(x_{2 i-1}, T x_{2 i-1}\right)+d_{q b}\left(x_{2 i}, S x_{2 i}\right)\right]+\varepsilon \\
& \leqslant \lambda d_{q b}\left(x_{2 i-1}, x_{2 i}\right)+\beta\left[d_{q b}\left(x_{2 i-1}, x_{2 i}\right)+d_{q b}\left(x_{2 i}, x_{2 i+1}\right)\right]+\varepsilon \\
& \leqslant(\lambda+\beta) d_{q b}\left(x_{2 i-1}, x_{2 i}\right)+\beta d_{q b}\left(x_{2 i}, x_{2 i+1}\right)+\varepsilon
\end{aligned}
$$

that is,

$$
d_{q b}\left(x_{2 i}, x_{2 i+1}\right) \leqslant k d_{q b}\left(x_{2 i-1}, x_{2 i}\right)+\frac{\varepsilon}{1-\beta} .
$$

It follows from (2.6) that

$$
d_{q b}\left(x_{2 i}, x_{2 i+1}\right) \leqslant k^{2 i} d_{q b}\left(x_{0}, x_{1}\right)+\frac{\varepsilon}{1-\beta} \sum_{p=0}^{2 i-1} k^{p}
$$

Now by (2.7), we get

$$
d_{q b}\left(x_{2 i}, x_{2 i+1}\right) \leqslant k^{2 i+1}(1-s k) r .
$$

Since $x_{2 i+1} \in S x_{2 i}$ and $S$ is $\alpha$-dominated mapping on $B_{q b}\left[x_{0}, r\right]$, we obtain $\alpha\left(x_{2 i}, x_{2 i+1}\right) \geqslant 1$. Now choose $\varepsilon>0$ such that

$$
k^{2 i+1} d_{q b}\left(x_{0}, x_{1}\right)+\frac{\varepsilon}{1-\beta} \sum_{p=0}^{2 i} k^{p} \leqslant k^{2 i+2}(1-s k) r .
$$

By Lemma 1.12, there exists $x_{2 i+2} \in T x_{2 i+1}$ such that

$$
d_{q b}\left(x_{2 i+1}, x_{2 i+2}\right) \leqslant H_{q b}\left(S x_{2 i}, T x_{2 i+1}\right)+\varepsilon .
$$

Using inequality (2.1), we have

$$
d_{q b}\left(x_{2 i+1}, x_{2 i+2}\right) \leqslant \lambda d_{q b}\left(x_{2 i}, x_{2 i+1}\right)+\beta\left[d_{q b}\left(x_{2 i}, S x_{2 i}\right)+d_{q b}\left(x_{2 i+1}, T x_{2 i+1}\right)\right]+\varepsilon
$$




$$
\begin{aligned}
& \leqslant \lambda d_{q b}\left(x_{2 i}, x_{2 i+1}\right)+\beta\left[d_{q b}\left(x_{2 i}, x_{2 i+1}\right)+d_{q b}\left(x_{2 i+1}, x_{2 i+2}\right)\right]+\varepsilon \\
& \leqslant(\lambda+\beta) d_{q b}\left(x_{2 i}, x_{2 i+1}\right)+\beta d_{q b}\left(x_{2 i+1}, x_{2 i+2}\right)+\varepsilon
\end{aligned}
$$

that is,

$$
d_{q b}\left(x_{2 i+1}, x_{2 i+2}\right) \leqslant k d_{q b}\left(x_{2 i}, x_{2 i+1}\right)+\frac{\varepsilon}{1-\beta},
$$

using (2.8) which further implies that

$$
d_{q b}\left(x_{2 i+1}, x_{2 i+2}\right) \leqslant k^{2 i+1} d_{q b}\left(x_{0}, x_{1}\right)+\frac{\varepsilon}{1-\beta} \sum_{p=0}^{2 i} k^{p} .
$$

Now by (2.9), we get

$$
d_{q b}\left(x_{2 i+1}, x_{2 i+2}\right) \leqslant k^{2 i+2}(1-s k) r .
$$

Thus, for some $j \in \mathbb{N}_{0}$ we have

$$
d_{q b}\left(x_{j}, x_{j+1}\right) \leqslant k^{j+1}(1-s k) r .
$$

Note that

$$
\begin{aligned}
d_{q b}\left(x_{0}, x_{j+1}\right) & \leqslant s d_{q b}\left(x_{0}, x_{1}\right)+s^{2} d_{q b}\left(x_{1}, x_{2}\right)+\cdots+s^{j} d_{q b}\left(x_{j-1}, x_{j}\right)+s^{j} d_{q b}\left(x_{j}, x_{j+1}\right) \\
& \leqslant s k(1-s k) r+s^{2} k^{2}(1-s k) r+\cdots+s^{j} k^{j}(1-s k) r+s^{j} k^{j+1}(1-s k) r \\
& \leqslant s k(1-s k) r+s^{2} k^{2}(1-s k) r+\cdots+s^{j} k^{j}(1-s k) r+s^{j+1} k^{j+1}(1-s k) r \\
& \leqslant s k(1-s k) r\left\{1+s k+(s k)^{2}+\cdots+(s k)^{j-2}+(s k)^{j-1}+(s k)^{j}\right\} \\
& \leqslant s k(1-s k) r\left\{1+s k+(s k)^{2}+\cdots+(s k)^{j-2}+(s k)^{j-1}+(s k)^{j}+\cdots\right\} \\
& \leqslant s k(1-s k) r\left\{\frac{1}{1-s k}\right\} \leqslant r
\end{aligned}
$$

which implies $x_{j+1} \in B_{q b}\left[x_{0}, r\right]$. Hence by induction, $x_{n} \in B_{q b}\left[x_{0}, r\right]$. Also $\alpha\left(x_{n}, x_{n+1}\right) \geqslant 1$ and inequality (2.10) can be written as

$$
d_{q b}\left(x_{n}, x_{n+1}\right) \leqslant k^{n+1}(1-s k) r
$$

for all $n \in \mathbb{N}_{0}$. Now

$$
\begin{aligned}
d_{q b}\left(x_{n}, x_{n+m}\right) & \leqslant s d_{q b}\left(x_{n}, x_{n+1}\right)+s^{2} d_{q b}\left(x_{n+1}, x_{n+2}\right)+\cdots+s^{m-1} d_{q b}\left(x_{n+m-1}, x_{n+m}\right) \\
& \leqslant s k^{n+1}(1-s k) r+s^{2} k^{n+2}(1-s k) r+\cdots+s^{m-1} k^{n+m}(1-s k) r \\
& \leqslant s k^{n+1}(1-s k) r+s^{2} k^{n+2}(1-s k) r+\cdots+s^{m} k^{n+m}(1-s k) r \\
& \leqslant s k^{n+1}(1-s k) r\left\{1+s k+(s k)^{2}+\cdots+(s k)^{m-2}+(s k)^{m-1}\right\} \\
& \leqslant s k^{n+1}(1-s k) r\left\{1+s k+(s k)^{2}+\cdots+(s k)^{m-2}+(s k)^{m-1}+\cdots\right\} \\
& \leqslant s k^{n+1}(1-s k) r \frac{1}{1-s k} \leqslant s k^{n+1} r
\end{aligned}
$$

Hence $\lim _{n, m \rightarrow \infty} d_{q b}\left(x_{n}, x_{n+m}\right)=0$ and we get that $\left\{x_{n}\right\}$ is a left K-Cauchy sequence in $\left(B_{q b}\left[x_{0}, r\right], d_{q b}\right)$. As every closed ball in a complete $d q b$-metric space is complete, so $\left(B_{q b}\left[x_{0}, r\right], d_{q b}\right)$ is left K-sequentially complete, there exists a point $x^{*} \in B_{q b}\left[x_{0}, r\right]$ such that

$$
\lim _{n \rightarrow \infty} d_{q b}\left(x_{n}, x^{*}\right)=\lim _{n \rightarrow \infty} d_{q b}\left(x^{*}, x_{n}\right)=0 .
$$

Suppose that condition (iii) (a) holds. Note that

$$
d_{\mathrm{qb}}\left(x^{*}, S x^{*}\right) \leqslant s\left\{d_{q b}\left(x^{*}, x_{2 n+1}\right)+d_{q b}\left(x_{2 n+1}, S x^{*}\right)\right\} \leqslant s\left\{d_{q b}\left(x^{*}, x_{2 n+1}\right)+H_{q b}\left(S x_{2 n}, S x^{*}\right)\right\} .
$$


Taking limit as $n \rightarrow \infty$ on both sides of above inequality, we obtain that $d_{q b}\left(x^{*}, S x^{*}\right) \leqslant 0$. That is, $\mathrm{d}_{\mathrm{qb}}\left(\mathrm{x}^{*}, \mathrm{~S} \mathrm{x}^{*}\right)=0$ and hence $\mathrm{x}^{*} \in \mathrm{S} \mathrm{x}^{*}$. Similarly,

$$
d_{q b}\left(x^{*}, T x^{*}\right) \leqslant s\left\{d_{q b}\left(x^{*}, x_{2 n+2}\right)+d_{q b}\left(x_{2 n+2}, T x^{*}\right)\right\} .
$$

Taking limit as $n \rightarrow \infty$ on both sides of above inequality, we obtain that $d_{q b}\left(x^{*}, T x^{*}\right) \leqslant 0$ gives that $x^{*} \in T x^{*}$. Next, we suppose that condition (iii) (b) holds. As $\alpha\left(x_{n}, x^{*}\right) \geqslant 1$, we have

$$
\begin{aligned}
\mathrm{d}_{\mathrm{qb}}\left(x^{*}, S x^{*}\right) & \leqslant s\left\{\mathrm{~d}_{\mathrm{qb}}\left(x^{*}, x_{2 n+2}\right)+\mathrm{d}_{\mathrm{qb}}\left(\mathrm{x}_{2 n+2}, S x^{*}\right)\right\} \\
& \leqslant s\left\{\mathrm{~d}_{\mathrm{qb}}\left(x^{*}, x_{2 n+2}\right)+\mathrm{H}_{\mathrm{qb}}\left(T x_{2 n+1}, S x^{*}\right)\right\} \\
& \leqslant s\left\{\mathrm{~d}_{\mathrm{qb}}\left(x^{*}, x_{2 n+2}\right)+\lambda \mathrm{d}_{\mathrm{qb}}\left(x_{2 n+1}, x^{*}\right)+\beta\left[\mathrm{d}_{\mathrm{qb}}\left(x_{2 n+1}, T x_{2 n+1}\right)+\mathrm{d}_{\mathrm{qb}}\left(x^{*}, S x^{*}\right)\right]\right\} \\
& \leqslant s\left\{\mathrm{~d}_{\mathrm{qb}}\left(x^{*}, x_{2 n+2}\right)+\lambda \mathrm{d}_{\mathrm{qb}}\left(x_{2 n+1}, x^{*}\right)+\beta\left[\mathrm{d}_{\mathrm{qb}}\left(x_{2 n+1}, x_{2 n+2}\right)+\mathrm{d}_{\mathrm{qb}}\left(x^{*}, S x^{*}\right)\right]\right\},
\end{aligned}
$$

which implies that

$$
(1-s \beta) d_{q b}\left(x^{*}, S x^{*}\right) \leqslant s d_{q b}\left(x^{*}, x_{2 n+2}\right)+s \lambda d_{q b}\left(x_{2 n+1}, x^{*}\right)+s \beta d_{q b}\left(x_{2 n+1}, x_{2 n+2}\right) .
$$

Taking limit as $n \rightarrow \infty$ on both sides of above inequality, we obtain $(1-s \beta) d_{q b}\left(x^{*}, S x^{*}\right) \leqslant 0$. This implies that $\mathrm{d}_{\mathrm{qb}}\left(x^{*}, S x^{*}\right)=0$. Hence $x^{*} \in S x^{*}$. Similarly, $x^{*} \in T x^{*}$. Since $S$ and $T$ are $\alpha$-dominated mappings on $\mathrm{B}_{\mathrm{qb}}\left[\mathrm{x}_{0}, \mathrm{r}\right]$, we have $\alpha\left(x^{*}, x^{*}\right) \geqslant 1$. Now if $S$ and $T$ are compact valued mappings, then by Lemma 1.14 with $\mu=1$, we have

$$
\mathrm{d}_{\mathrm{qb}}\left(x^{*}, x^{*}\right) \leqslant \mathrm{H}_{\mathrm{qb}}\left(S x^{*}, T x^{*}\right) \leqslant \lambda \mathrm{d}_{\mathrm{qb}}\left(x^{*}, x^{*}\right)+\beta\left[\mathrm{d}_{\mathrm{qb}}\left(x^{*}, S x^{*}\right)+\mathrm{d}_{\mathrm{qb}}\left(x^{*}, T x^{*}\right)\right],
$$

which implies that $(1-\lambda-2 \beta) d_{q b}\left(x^{*}, x^{*}\right) \leqslant 0$ and hence $d_{q b}\left(x^{*}, x^{*}\right)=0$.

\section{Remark 2.2.}

(i) Mappings $S$ and T satisfying the contractive conditions (2.1) and (2.2) are not necessarily continuous.

(ii) The result also holds for the right K-sequentially complete dq b-metric space.

(iii) Mappings $S$ and $T$ satisfying the contractive condition (2.1) are Suzuki type contraction mappings on a closed ball. Indeed, if for any $x, y \in B_{q b}\left[x_{0}, r\right]$ with $\frac{1}{2} \min \left\{d_{q b}(x, S x), d_{q b}(y, T y)\right\} \leqslant d_{q b}(x, y)$, we have

$$
H_{q b}(S x, T y) \leqslant \lambda d_{q b}(x, y)+\beta\left[d_{q b}(x, S x)+d_{q b}(y, T y)\right],
$$

and define $\alpha(x, y)=d_{q b}(x, y)-\frac{1}{2} \min \left\{d_{q b}(x, S x), d_{q b}(y, T y)\right\}+1$ for all $x, y \in B_{q b}\left[x_{0}, r\right]$, then we have $\alpha(x, y) \geqslant 1$, which further implies that

$$
H_{q b}(S x, T y) \leqslant \lambda d_{q b}(x, y)+\beta\left[d_{q b}(x, S x)+d_{q b}(y, T y)\right] .
$$

The following example supports Remark 2.2 (i).

Example 2.3. Let $X=\mathbb{R}^{+}$. Define $d_{q b}: X \times X \rightarrow \mathbb{R}^{+}$by $d_{q b}(x, y)=|x-y|^{2}$. Note that $\left(X, d_{q b}\right)$ is a left $\mathrm{K}$-sequentially complete $\mathrm{dq} b$-metric space with $s=2$. If $x_{0}=\frac{1}{4}$ and $r=\frac{1}{4}$, then $B_{q b}\left[x_{0}, r\right]=\left[0, \frac{1}{2}\right]$. Define $S, T: X \rightarrow \mathrm{CB}(X)$ and $\alpha: X \times X \rightarrow \mathbb{R}^{+}$by

$$
\begin{gathered}
S x= \begin{cases}{\left[\frac{x}{4}, \frac{x}{3}\right],} & \text { if } x \in\left[0, \frac{1}{2}\right], \\
{[x, x+1],} & \text { otherwise, }\end{cases} \\
T x= \begin{cases}{\left[\frac{x}{6}, \frac{x}{3}\right],} & \text { if } x \in\left[0, \frac{1}{2}\right], \\
{[x+1, x+3],} & \text { otherwise, }\end{cases}
\end{gathered}
$$

and $\alpha(x, y)=1$ if $x, y \in\left[0, \frac{1}{2}\right]$ and $\alpha(x, y)=\frac{1}{3}$ otherwise. Obviously $S$ and $T$ are $\alpha$-dominated mappings on $B_{q b}\left[x_{0}, r\right]$ but not on $X-B_{q b}\left[x_{0}, r\right]$. For $x, y \in B_{q b}\left[x_{0}, r\right]$, we have

$$
\mathrm{H}_{\mathrm{qb}}(\mathrm{Sx}, \mathrm{Ty})=\mathrm{H}_{\mathrm{qb}}\left(\left[\frac{x}{4}, \frac{x}{3}\right],\left[\frac{y}{6}, \frac{y}{3}\right]\right)=\left|\frac{x}{3}-\frac{y}{3}\right|^{2}=\frac{1}{9}|x-y|^{2} \leqslant \frac{1}{6} \mathrm{~d}_{\mathrm{qb}}(x, y)+\frac{1}{8}\left[\mathrm{~d}_{\mathrm{qb}}(x, S x)+\mathrm{d}_{\mathrm{qb}}(y, T y)\right] .
$$


Hence, $S$ and $T$ satisfy the inequality (2.1) and similarly, we can show that $S$ and $T$ satisfy (2.2) on $B_{q b}\left[x_{0}, r\right]$ with $k=\frac{1}{3}, \lambda=\frac{1}{6}$, and $\beta=\frac{1}{8}$. Now consider a sequence $x_{n}=\frac{1}{2}+\frac{1}{n}$ in $X$. Then there exists $x=\frac{1}{2}$ such that $\lim _{n \rightarrow \infty} d_{q b}\left(x_{n}, x\right)=0$ but

$$
\lim _{n \rightarrow \infty} H_{q b}\left(S x_{n}, S x\right)=\lim _{n \rightarrow \infty} H_{q b}\left(\left[\frac{1}{2}+\frac{1}{n}, \frac{3}{2}+\frac{1}{n}\right],\left[\frac{1}{8}, \frac{1}{6}\right]\right)=\lim _{n \rightarrow \infty}\left|\frac{4}{3}+\frac{1}{n}\right|^{2} \neq 0 .
$$

Therefore, $S$ is not continuous at $\frac{1}{2}$ and similarly we can show that $\mathrm{T}$ is not continuous at $\frac{1}{2}$. For $x_{0}=\frac{1}{4}$ and $\frac{1}{12}=x_{1} \in S x_{0}=\left[\frac{1}{16}, \frac{1}{12}\right]$, we have $d_{q b}\left(\frac{1}{4}, \frac{1}{12}\right)=\left|\frac{1}{4}-\frac{1}{12}\right|^{2} \leqslant k(1-s k) r$. For any sequence $\left\{x_{n}\right\}$ in $B_{q b}\left[x_{0}, r\right]$ with $\alpha\left(x_{n}, x_{n+1}\right) \geqslant 1$ for all $n \in \mathbb{N}_{0}$ and $x_{n} \rightarrow x \in B_{q b}\left[x_{0}, r\right]$ as $n \rightarrow \infty$, we obtain that $\alpha\left(x_{n}, x\right) \geqslant 1$ for all $n \in \mathbb{N}_{0}$. Thus all conditions of Theorem 2.1 are satisfied. Therefore, $x^{*}=0$ is a common fixed point of $S$ and $T$ in $B_{q b}\left[x_{0}, r\right]$, then we have $d_{q b}\left(x^{*}, x^{*}\right)=0$ as $S x$ and $T x$ are compact sets for any $x \in X$.

Example 2.4. Let $X=\mathbb{R}^{+}$and $d_{q b}(x, y)=|x-y|^{2}+\frac{x^{2}}{36}$ for all $x, y \in X$. Define the mappings $S, T: X \rightarrow$ $\mathrm{CB}(\mathrm{X})$ by

$$
S x= \begin{cases}\left\{\frac{2 x}{3}\right\}, & \text { if } x \in[0,1] \\ \{x+1\}, & \text { if } x \in(1, \infty),\end{cases}
$$

and

$$
\mathrm{Tx}= \begin{cases}{\left[0, \frac{x}{3}\right],} & \text { if } x \in[0,1] \\ {[x, 2 x],} & \text { if } x \in(1, \infty) .\end{cases}
$$

Note that $\left(X, d_{q b}\right)$ is a left $K$-sequentially complete $d q b$-metric with $s=2$. If $x_{0}=\frac{1}{2}$ and $r=\frac{1}{2}$, then $\mathrm{B}_{\mathrm{qb}}\left[\mathrm{x}_{0}, \mathrm{r}\right]=[0,1]$. Define $\alpha: \mathrm{X} \times \mathrm{X} \rightarrow \mathbb{R}^{+}$by

$$
\alpha(x, y)= \begin{cases}\frac{1}{|x-y|}, & \text { if } x, y \in[0,1] \text { and } x \neq y \\ 1, & \text { if } x, y \in[0,1] \text { and } x=y \\ \frac{1}{3}, & \text { otherwise }\end{cases}
$$

Clearly $S, T$ are $\alpha$-dominated mappings on $B_{\mathrm{qb}}\left[\mathrm{x}_{0}, \mathrm{r}\right]$ but not on $\mathrm{X}-\mathrm{B}_{\mathrm{qb}}\left[\mathrm{x}_{0}, \mathrm{r}\right]$ and satisfy the inequalities (2.1) and (2.2) on $B_{q b}\left[x_{0}, r\right]$ with $k=\frac{2}{5}, \lambda=\frac{1}{6}$, and $\beta=\frac{1}{6}$. Also, for $x_{0}=\frac{1}{2}$ and $\frac{1}{3}=x_{1} \in S x_{0}=\left\{\frac{2 x_{0}}{3}\right\}$, we have $d_{q b}\left(\frac{1}{2}, \frac{1}{3}\right)=\frac{5}{144} \leqslant \frac{2}{5}\left(1-2\left(\frac{2}{5}\right)\right) \frac{1}{2}=\frac{1}{25}=k(1-s k)$. For any sequence $\left\{x_{n}\right\}$ in $B_{q b}\left[x_{0}, r\right]$ with $\alpha\left(x_{n}, x_{n+1}\right) \geqslant 1$ for all $n \in \mathbb{N}_{0}$ and $x_{n} \rightarrow x \in B_{q b}\left[x_{0}, r\right]$ as $n \rightarrow \infty$, we obtain that $\alpha\left(x_{n}, x\right) \geqslant 1$ for all $n \in \mathbb{N}_{0}$. Thus all the conditions of Theorem 2.1 are satisfied. Hence $x^{*}=0$ is the common fixed point of $S$ and $T$ in $B_{q b}\left[x_{0}, r\right]$. As $S x$ and $T x$ are compact sets for each $x \in X$, we have $d_{q b}(0,0)=0$.

Corollary 2.5. Let $\left(\mathrm{X}, \mathrm{d}_{\mathrm{qb}}\right)$ be a left $\mathrm{K}$-sequentially complete $d q$ b-metric space with $\mathrm{x}_{0} \in \mathrm{X}$ and $\alpha: \mathrm{X} \times \mathrm{X} \rightarrow \mathbb{R}^{+}$. Suppose that $\mathrm{S}: \mathrm{X} \rightarrow \mathrm{CB}(\mathrm{X})$ is $\alpha$-dominated mapping on $\mathrm{B}_{\mathrm{qb}}\left[\mathrm{x}_{0}, \mathrm{r}\right]$ and for any $\mathrm{x}, \mathrm{y} \in \mathrm{B}_{\mathrm{qb}}\left[\mathrm{x}_{0}, \mathrm{r}\right]$ with $\alpha(\mathrm{x}, \mathrm{y}) \geqslant 1$, we have

$$
H_{q b}(S x, S y) \leqslant \lambda d_{q b}(x, y)+\beta\left[d_{q b}(x, S x)+d_{q b}(y, S y)\right],
$$

where $0 \leqslant \lambda+2 \beta<1$. If there exists $x_{1} \in S x_{0}$ such that

$$
d_{\mathrm{qb}}\left(x_{0}, x_{1}\right) \leqslant k(1-s k) r,
$$

where $\mathrm{k}=\frac{\lambda+\beta}{1-\beta}$ and $\mathrm{sk}<1$, then $\mathrm{S}$ has a fixed point $\mathrm{x}^{*} \in \mathrm{B}_{\mathrm{qb}}\left[\mathrm{x}_{0}, \mathrm{r}\right]$ provided that $\mathrm{S}$ is continuous or for any sequence $\left\{x_{n}\right\}$ in $B_{\mathrm{qb}}\left[x_{0}, r\right]$ with $\alpha\left(x_{n}, x_{n+1}\right) \geqslant 1$ for all $n \in \mathbb{N}_{0}$ and $x_{n} \rightarrow x \in B_{q b}\left[x_{0}, r\right]$, we have $\alpha\left(x_{n}, x\right) \geqslant 1$ for all $\mathrm{n} \in \mathbb{N}_{0}$. Moreover, if $\mathrm{S}$ is compact valued, then $\mathrm{d}_{\mathrm{qb}}\left(\mathrm{x}^{*}, \mathrm{x}^{*}\right)=0$.

Proof. If $\mathrm{S}=\mathrm{T}$ in Theorem 2.1, we obtain $\mathrm{x}^{*} \in \mathrm{B}_{\mathrm{qb}}\left[\mathrm{x}_{0}, \mathrm{r}\right]$ such that $\mathrm{x}^{*} \in \mathrm{S} \mathrm{x}^{*}$ and $\mathrm{d}_{\mathrm{qb}}\left(\mathrm{x}^{*}, \mathrm{x}^{*}\right)=0$.

Corollary 2.6. Let $\left(\mathrm{X}, \mathrm{d}_{\mathrm{q}}\right)$ be a left $\mathrm{K}$-sequentially complete $d q$ b-metric space, $\mathrm{x}_{0} \in \mathrm{X}$, and $\alpha: \mathrm{X} \times \mathrm{X} \rightarrow \mathbb{R}^{+}$. Suppose that $\mathrm{S}, \mathrm{T}: \mathrm{X} \rightarrow \mathrm{CB}(\mathrm{X})$ are $\alpha$-dominated mappings on $\mathrm{B}_{\mathrm{qb}}\left[\mathrm{x}_{0}, \mathrm{r}\right]$ such that for any $\mathrm{x}, \mathrm{y} \in \mathrm{B}_{\mathrm{qb}}\left[\mathrm{x}_{0}, \mathrm{r}\right]$ with $\alpha(x, y) \geqslant 1$, we have 


$$
\alpha(x, y) H_{q b}(S x, T y) \leqslant \lambda d_{q b}(x, y)+\beta\left[d_{q b}(x, S x)+d_{q b}(y, T y)\right]
$$

and

$$
\alpha(x, y) H_{q b}(T x, S y) \leqslant \lambda d_{q b}(x, y)+\beta\left[d_{q b}(x, T x)+d_{q b}(y, S y)\right],
$$

where $0 \leqslant \lambda+2 \beta<1$. If there exists $\mathrm{x}_{1} \in \mathrm{S} \mathrm{x}_{0}$ such that

$$
d_{q b}\left(x_{0}, x_{1}\right) \leqslant k(1-s k) r,
$$

where $\mathrm{k}=\frac{\lambda+\beta}{1-\beta}$ and $\mathrm{sk}<1$, then $\mathrm{S}$ and $\mathrm{T}$ have a common fixed point $\mathrm{x}^{*} \in \mathrm{B}_{\mathrm{qb}}\left[\mathrm{x}_{0}, \mathrm{r}\right]$ provided that either $\mathrm{S}, \mathrm{T}$ are continuous or for any sequence $\left\{x_{n}\right\}$ in $X$ with $\alpha\left(x_{n}, x_{n+1}\right) \geqslant 1$ for all $n \in \mathbb{N}_{0}$ and $x_{n} \rightarrow x \in B_{q b}\left[x_{0}, r\right]$, we have $\alpha\left(x_{n}, x\right) \geqslant 1$ for all $\mathrm{n} \in \mathbb{N}_{0}$. Moreover, if $S$ and $T$ are compact valued, then $\mathrm{d}_{\mathrm{qb}}\left(\mathrm{x}^{*}, \mathrm{x}^{*}\right)=0$.

In Theorem 2.1, the condition (2.3) restricts the conditions (2.1) and (2.2) to $B_{q b}\left[x_{0}, r\right]$. If we relax the condition (2.3) and consider the conditions (2.1) and (2.2) for all elements $x, y$ in $X$, then we have the following result.

Theorem 2.7. Let $\left(\mathrm{X}, \mathrm{d}_{\mathrm{qb}}\right)$ be a left $\mathrm{K}$-sequentially complete $d q$ b-metric space and $\alpha: \mathrm{X} \times \mathrm{X} \rightarrow \mathbb{R}^{+}$. Suppose that $\mathrm{S}, \mathrm{T}: \mathrm{X} \rightarrow \mathrm{CB}(\mathrm{X})$ are $\alpha$-dominated mappings on $\mathrm{X}$ such that for any $\mathrm{x}, \mathrm{y} \in \mathrm{X}$ with $\alpha(\mathrm{x}, \mathrm{y}) \geqslant 1$, we have

$$
H_{q b}(S x, T y) \leqslant \lambda d_{q b}(x, y)+\beta\left[d_{q b}(x, S x)+d_{q b}(y, T y)\right]
$$

and

$$
H_{q b}(T x, S y) \leqslant \lambda d_{q b}(x, y)+\beta\left[d_{q b}(x, T x)+d_{q b}(y, S y)\right]
$$

where $0 \leqslant \lambda+2 \beta<1$. If either $S$ and $T$ are continuous or for any sequence $\left\{x_{n}\right\}$ in $X$ with $\alpha\left(x_{n}, x_{n+1}\right) \geqslant 1$ for all $\mathrm{n} \in \mathbb{N}_{0}$ and $x_{n} \rightarrow x \in X$, we have $\alpha\left(x_{n}, x\right) \geqslant 1$ for all $\mathrm{n} \in \mathbb{N}_{0}$. Then $\mathrm{S}$ and $\mathrm{T}$ have a common fixed point $x^{*} \in X$. Moreover, if $\mathrm{S}$ and $\mathrm{T}$ are compact valued, then $\mathrm{d}_{\mathrm{qb}}\left(\mathrm{x}^{*}, \mathrm{x}^{*}\right)=0$.

Proof. Fix $x_{0} \in X$ and choose $r>0$ such that

$$
\mathrm{d}_{\mathrm{qb}}\left(\mathrm{x}_{0}, \mathrm{x}_{1}\right) \leqslant \mathrm{k}(1-\mathrm{sk}) \mathrm{r}
$$

for $x_{1} \in S x_{0}$, where $k=\frac{\lambda+\beta}{1-\beta}$ and $s k<1$. The result then follows from Theorem 2.1.

Example 2.8. If $X=Q^{+}$and $d_{q b}(x, y)=|x-y|^{2}+\frac{|x|}{2}+\frac{|y|}{3}$, then $\left(X, d_{q b}\right)$ is a left $K$-sequentially complete $\mathrm{dq} b$-metric space with $s=2$. Define the mappings $S, T: X \rightarrow C B(X)$ by

$$
S x= \begin{cases}\left\{x \in \mathbb{Q}^{+} \mid 3<x^{2}<5\right\}, & \text { if } x \in \mathbb{Q}^{+}-\{0\}, \\ \{x\}, & \text { if } x=0,\end{cases}
$$

and

$$
T x= \begin{cases}\left\{x \in \mathbb{Q}^{+} \mid 4 \leqslant x^{2}<5\right\}, & \text { if } x \in \mathbb{Q}^{+}-\{0\}, \\ \left\{\frac{x}{2}\right\}, & \text { if } x=0 .\end{cases}
$$

Define $\alpha: X \times X \rightarrow \mathbb{R}^{+}$as $\alpha(x, y)=\ln (x+y+e)$ if $x, y \in \mathbb{Q}^{+}-\{0\}$ and $\alpha(x, y)=1$ otherwise. Clearly, the mappings $S, T$ are $\alpha$-dominated mappings on $X$ and satisfy inequalities (2.11) and (2.12) for any $x, y \in X$. Moreover, for any sequence $\left\{x_{n}\right\}$ in $X$ with $\alpha\left(x_{n}, x_{n+1}\right) \geqslant 1$ for all $n \in \mathbb{N}_{0}$ and $x_{n} \rightarrow x$, we have $\alpha\left(x_{n}, x\right) \geqslant 1$ for all $n \in \mathbb{N}_{0}$. Thus, all conditions of Theorem 2.7 are satisfied and we obtain $x^{*}$ in $X$ such that $x^{*} \in S x^{*} \cap T x^{*}=\left\{x^{*} \in \mathbb{Q}^{+} \mid 4 \leqslant\left(x^{*}\right)^{2}<5\right\} \cup\{0\}$. As $S$ and $T$ are not compact valued, $\mathrm{d}_{\mathrm{qb}}\left(x^{*}, x^{*}\right)=0$ does not hold for some $x^{*} \in S x^{*} \cap \mathrm{T} x^{*}$. For example, $2 \in \mathbb{Q}^{+}-\{0\}$ and $2 \in S 2 \cap \mathrm{T} 2=\left\{y \in \mathbb{Q}^{+} \mid 4 \leqslant y^{2}<5\right\}$ but $\mathrm{d}_{\mathrm{qb}}(2,2)=|2-2|^{2}+\frac{|2|}{2}+\frac{|2|}{3}=1+\frac{2}{3} \neq 0$. 
Corollary 2.9. Let $\left(\mathrm{X}, \mathrm{d}_{\mathrm{qb}}\right)$ be a left $\mathrm{K}$-sequentially complete $d q$ b-metric space, and $\alpha: \mathrm{X} \times \mathrm{X} \rightarrow \mathbb{R}^{+}$. Suppose that $\mathrm{S}: \mathrm{X} \rightarrow \mathrm{CB}(\mathrm{X})$ is $\alpha$-dominated mapping on $\mathrm{X}$ such that for any $\mathrm{x}, \mathrm{y} \in \mathrm{X}$ with $\alpha(\mathrm{x}, \mathrm{y}) \geqslant 1$, we have

$$
\mathrm{H}_{\mathrm{qb}}(S x, S y) \leqslant \lambda \mathrm{d}_{\mathrm{qb}}(x, y)+\beta\left[\mathrm{d}_{\mathrm{qb}}(x, S x)+\mathrm{d}_{\mathrm{qb}}(y, S y)\right],
$$

where $0 \leqslant \lambda+2 \beta<1$. If $S$ is continuous or for any sequence $\left\{x_{n}\right\}$ in $X$ with $\alpha\left(x_{n}, x_{n+1}\right) \geqslant 1$ for all $n \in \mathbb{N}_{0}$ and $x_{n} \rightarrow x \in X$, we have $\alpha\left(x_{n}, x\right) \geqslant 1$ for all $n \in \mathbb{N}_{0}$. Then $S$ has a fixed point $x^{*}$ in $X$. Moreover, if $S$ is compact valued, then $\mathrm{d}_{\mathrm{qb}}\left(x^{*}, x^{*}\right)=0$.

Proof. The result follows from Theorem 2.7.

Corollary 2.10. Let $\left(\mathrm{X}, \mathrm{d}_{\mathrm{qb}}\right)$ be a left $\mathrm{K}$-sequentially complete $d q$ b-metric space with $\mathrm{x}_{0} \in \mathrm{X}$ and $\mathrm{S}, \mathrm{T}: \mathrm{X} \rightarrow \mathrm{CB}(\mathrm{X})$. Suppose that for any $\mathrm{x}, \mathrm{y}$ in $\mathrm{B}_{\mathrm{qb}}\left[\mathrm{x}_{0}, \mathrm{r}\right]$, we have

$$
H_{q b}(S x, T y) \leqslant \lambda d_{q b}(x, y)+\beta\left[d_{q b}(x, S x)+d_{q b}(y, T y)\right]
$$

and

$$
\mathrm{H}_{\mathrm{qb}}(T x, S y) \leqslant \lambda \mathrm{d}_{\mathrm{qb}}(x, y)+\beta\left[\mathrm{d}_{\mathrm{qb}}(x, T x)+\mathrm{d}_{\mathrm{qb}}(\mathrm{y}, \mathrm{Sy})\right],
$$

where $\lambda+2 \beta \in[0,1)$. If there exists $x_{1} \in S x_{0}$ such that

$$
\mathrm{d}_{\mathrm{qb}}\left(x_{0}, x_{1}\right) \leqslant k(1-s k) r,
$$

where $\mathrm{k}=\frac{\lambda+\beta}{1-\beta}$ and $\mathrm{sk}<1$, then $\mathrm{S}$ and $\mathrm{T}$ have a common fixed point $\mathrm{x}^{*}$ in $\mathrm{B}_{\mathrm{qb}}\left[\mathrm{x}_{0}, \mathrm{r}\right]$. Moreover, if $\mathrm{S}$ and $\mathrm{T}$ are compact valued, then $\mathrm{d}_{\mathrm{qb}}\left(\mathrm{x}^{*}, \mathrm{x}^{*}\right)=0$.

Proof. If we define $\alpha(x, y)=1$ for all $x, y$ in $B_{q b}\left[x_{0}, r\right]$, then the result follows from Theorem 2.1.

Corollary 2.11. Let $\left(\mathrm{X}, \mathrm{d}_{\mathrm{qb}}\right)$ be a left $\mathrm{K}$-sequentially complete $d q$-metric space with $\mathrm{x}_{0} \in \mathrm{X}$ and $\mathrm{S}: \mathrm{X} \rightarrow \mathrm{CB}(\mathrm{X})$. Suppose that for any $\mathrm{x}, \mathrm{y}$ in $\mathrm{B}_{\mathrm{qb}}\left[\mathrm{x}_{0}, \mathrm{r}\right]$, we have

$$
H_{q b}(S x, S y) \leqslant \lambda d_{q b}(x, y)+\beta\left[d_{q b}(x, S x)+d_{q b}(y, S y)\right],
$$

where $\lambda+2 \beta \in[0,1)$. If there exists $x_{1} \in S x_{0}$ such that

$$
d_{\mathrm{qb}}\left(x_{0}, x_{1}\right) \leqslant k(1-s k) r,
$$

where $\mathrm{k}=\frac{\lambda+\beta}{1-\beta}$ and $\mathrm{sk}<1$, then $\mathrm{S}$ has a fixed point $\mathrm{x}^{*}$ in $\mathrm{B}_{\mathrm{qb}}\left[\mathrm{x}_{0}, \mathrm{r}\right]$. Moreover, if $\mathrm{S}$ is compact valued, then $\mathrm{d}_{\mathrm{qb}}\left(x^{*}, x^{*}\right)=0$.

Example 2.12. If $X=\mathbb{R}^{+}$and $d_{q b}(x, y)=|x-y|^{2}$, then $\left(X, d_{q b}\right)$ is a left $K$-sequentially complete dq b-metric space with $s=2$. Define the mapping $S: X \rightarrow C B(X)$ by

$$
S x= \begin{cases}{\left[0, \frac{x}{3}\right],} & \text { if } x \in\left[0, \frac{1}{2}\right] \\ {\left[\frac{2 x}{3}, \frac{3 x}{4}\right],} & \text { if } x \in\left(\frac{1}{2}, \infty\right)\end{cases}
$$

If $x_{0}=\frac{1}{4}, \frac{1}{12}=x_{1} \in S x_{0}=\left[0, \frac{1}{12}\right]$ and $r=\frac{1}{4}$, then $B_{q b}\left[x_{0}, r\right]=\left[0, \frac{1}{2}\right]$. Define $\alpha: X \times X \rightarrow \mathbb{R}^{+}$as $\alpha(x, y)=2$ if $x, y \in[0,1]$ and $\alpha(x, y)=\frac{4}{3}$ otherwise. Clearly, the mapping $S$ is $\alpha$-dominated mapping on $X$. For $\lambda=\beta=\frac{1}{9}$ and $k=\frac{1}{4}$, the mapping $S$ satisfies inequality (2.13) for any $x, y \in X$. Thus, all conditions of Corollary 2.11 are satisfied and $S$ has a fixed point $x^{*}=0$. Also, $d_{q b}(0,0)=0$.

Corollary 2.13. Let $\left(\mathrm{X}, \mathrm{d}_{\mathrm{qb}}\right)$ be a left $\mathrm{K}$-sequentially complete $d q$-metric space and $\mathrm{S}: \mathrm{X} \rightarrow \mathrm{CB}(\mathrm{X})$. If for any $x, y$ in $X$, we have

$$
\mathrm{H}_{\mathrm{qb}}(S x, S y) \leqslant \lambda d_{\mathrm{qb}}(x, y)+\beta\left[\mathrm{d}_{\mathrm{qb}}(x, S x)+\mathrm{d}_{\mathrm{qb}}(\mathrm{y}, \mathrm{Sy})\right],
$$

where $\lambda+2 \beta \in[0,1)$, then $S$ has a fixed point $x^{*}$ in $X$. Moreover, if $S$ is compact valued, then $\mathrm{d}_{\mathrm{qb}}\left(\mathrm{x}^{*}, \mathrm{x}^{*}\right)=0$. 
Example 2.14. If $X=\mathbb{R}^{+}$and $d_{q b}(x, y)=|x-y|^{2}$, then $\left(X, d_{q b}\right)$ is a left K-sequentially complete dq b-metric space with $s=2$. Define the mapping $S: X \rightarrow C B(X)$ by

$$
S x= \begin{cases}{\left[0, \frac{x}{4}\right],} & \text { if } x \in[0,1] \\ \left\{\frac{x}{8}\right\}, & \text { if } x \in(1, \infty)\end{cases}
$$

Define $\alpha: X \times X \rightarrow \mathbb{R}^{+}$as $\alpha(x, y)=2$ for any $x, y \in X$. Clearly, the mapping $S$ is $\alpha$-dominated mapping on $X$. For $\lambda=\frac{1}{9}, \beta=\frac{2}{9}$, and $k=\frac{3}{7}$, the mapping $S$ satisfies inequality (2.14) for any $x, y \in X$. Thus, all conditions of Corollary 2.13 are satisfied and $S$ has a fixed point $\chi^{*}=0$. Also, $d_{q b}(0,0)=0$.

Remark 2.15. Note that, dislocated quasi metric, dislocated b-metric, quasi b-metric, partial b-metric, bmetric, dislocated metric, quasi metric, partial metric, and ordinary metric versions of our results are also new in the literature.

\section{Applications on a complete dq b-metric space endowed with an arbitrary binary relation}

In this section, we study the necessary conditions for existence of common fixed point of mappings defined on left $\mathrm{K}$-sequentially complete dq b-metric spaces endowed with an arbitrary binary relation $\mathcal{R}$.

Let us recall the following definitions and known results.

Definition $3.1([22,31])$. Let $X$ be a nonempty set. A subset $\mathcal{R}$ of $X^{2}$ is called a binary relation on $X$. For

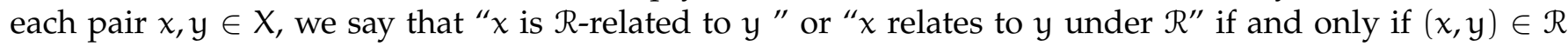
and $(x, y) \notin \mathcal{R}$ means that " $x$ is not $\mathcal{R}$-related to $y$ " or " $x$ does not relate to $y$ under $\mathcal{R}$ ".

Notice that $X^{2}$ and $\emptyset$ being subsets of $X^{2}$ are binary relations on $X$, which are called universal relation (or full relation) and empty relation, respectively.

Definition $3.2([1])$. Let $\mathcal{R}$ be a binary relation defined on a nonempty set $X$. Then any pair of points $x, y$ in $X$ is called $\mathcal{R}$-comparative if either $(x, y) \in \mathcal{R}$ or $(y, x) \in \mathcal{R}$, which is written as $[x, y] \in \mathcal{R}$.

Definition 3.3 ([22]). A binary relation $\mathcal{R}$ defined on a nonempty set $X$ is called (i) reflexive if $(x, x) \in \mathcal{R}$ $\forall x \in X$; (ii) irreflexive if $(x, x) \notin \mathcal{R}$ for some $x \in X$; (iii) symmetric if $(x, y) \in \mathcal{R}$ implies $(y, x) \in \mathcal{R} \forall x, y \in X$; (iv) antisymmetric if $(x, y) \in \mathcal{R}$ and $(y, x) \in \mathcal{R}$ imply $x=y, \forall x, y \in X ;(v)$ transitive if $(x, y) \in \mathcal{R}$ and $(y, z) \in \mathcal{R}$ imply $(x, z) \in \mathcal{R} \forall x, y, z \in X$; (vi) preorder if $\mathcal{R}$ is reflexive and transitive; (vii) partial order if $\mathcal{R}$ is reflexive, antisymmetric, and transitive.

We set

$$
\nabla \mathcal{R}=\{(x, y) \in X \times X:[x, y] \in \mathcal{R}\} .
$$

Definition 3.4 ([1]). Let $X$ be a nonempty set and $\mathcal{R}$ a binary relation on $X$. A sequence $\left\{x_{n}\right\} \subset X$ is called $\mathcal{R}$-preserving if $\left(x_{n}, x_{n+1}\right) \in \mathcal{R} \forall n \in \mathbb{N}_{0}$.

Definition 3.5. Let $X$ be a nonempty set, and $\mathcal{R}$ a binary relation on $X$. A multivalued mapping $T: X \rightarrow$ $\mathrm{N}(\mathrm{X})$ is called $\mathcal{R}$-dominated mapping on $X$ if for each $x \in X$, we have $(x, u) \in \mathcal{R}$ for any $u \in T x$. In particular $T$ is $\mathcal{R}$-dominated mapping on $A \subseteq X$ if for each $x \in A$, we have $(x, u) \in \mathcal{R}$ for any $u \in T x$.

Theorem 3.6. Let $\left(\mathrm{X}, \mathrm{d}_{\mathrm{q}}, \mathcal{R}\right)$ be a left $\mathrm{K}$-sequentially complete $d q$-metric space endowed with $\mathcal{R}$ with $\mathrm{x}_{0} \in \mathrm{X}$ and $\mathrm{S}, \mathrm{T}: \mathrm{X} \rightarrow \mathrm{CB}(\mathrm{X})$. Suppose that the following conditions hold:

(i) $\mathrm{S}$ and $\mathrm{T}$ are $\mathcal{R}$-dominated mappings on $\mathrm{B}_{\mathrm{qb}}\left[\mathrm{x}_{0}, \mathrm{r}\right]$;

(ii) there exist some constants $\lambda$, $\beta$ satisfying $0 \leqslant \lambda+2 \beta<1$ such that for any $(x, y) \in B_{q b}\left[x_{0}, r\right] \times B_{q b}\left[x_{0}, r\right] \cap$ $\nabla \mathcal{R}$, we have

$$
H_{q b}(S x, T y) \leqslant \lambda d_{q b}(x, y)+\beta\left[d_{q b}(x, S x)+d_{q b}(y, T y)\right]
$$

and

$$
H_{q b}(T x, S y) \leqslant \lambda d_{q b}(x, y)+\beta\left[d_{q b}(x, T x)+d_{q b}(y, S y)\right]
$$


(iii) there exists $x_{1} \in S x_{0}$ such that

$$
d_{q b}\left(x_{0}, x_{1}\right) \leqslant k(1-s k) r
$$

holds, where $\mathrm{k}=\frac{\lambda+\beta}{1-\beta}$ and $\mathrm{sk}<1$;

(iv) either $\mathrm{S}$ and $\mathrm{T}$ are continuous or for any sequence $\left\{x_{\mathrm{n}}\right\}$ in $\mathrm{B}_{\mathrm{qb}}\left[\mathrm{x}_{0}, \mathrm{r}\right]$ such that $\left\{x_{\mathrm{n}}\right\}$ is $\mathcal{R}$-preserving and $x_{n} \rightarrow x \in B_{\mathrm{qb}}\left[x_{0}, r\right]$, we have $\left(x_{n}, x\right) \in \mathcal{R}$ for all $n \in \mathbb{N}_{0}$.

Then $\mathrm{S}$ and $\mathrm{T}$ have a common fixed point $\mathrm{x}^{*} \in \mathrm{B}_{\mathrm{qb}}\left[\mathrm{x}_{0}, \mathrm{r}\right]$. Moreover, if $\mathrm{S}$ and $\mathrm{T}$ are compact valued, then $\mathrm{d}_{\mathrm{qb}}\left(x^{*}, x^{*}\right)=0$.

Proof. If we define the mapping $\alpha: X \times X \rightarrow \mathbb{R}^{+}$by

$$
\alpha(x, y)= \begin{cases}1, & \text { if }(x, y) \in \nabla \mathcal{R} \\ 0, & \text { otherwise }\end{cases}
$$

then, all the conditions of Theorem 2.1 are satisfied and hence we have the result.

Remark 3.7. Similar result as the above theorem can be established if the binary relation $\mathcal{R}$ is $\mathcal{R}$-reversing.

Example 3.8. Let $X=[0,1]$ and $d_{q b}: X \times X \rightarrow \mathbb{R}^{+}$be defined by $d_{q b}(x, y)=|x-y|^{2}$. It is clear that $\left(X, d_{q b}\right)$ is a left K-sequentially complete $d q b$-metric with $s=2$. As $d_{q b}(1,0) \not \leq d_{q b}\left(1, \frac{1}{2}\right)+d_{q b}\left(\frac{1}{2}, 0\right)$, so $\left(X, d_{q b}\right)$ is not a metric space. Define the mappings $S, T: X \rightarrow C B(X)$ by

$$
S x= \begin{cases}\left\{0, \frac{x}{3}\right\}, & \text { if } x \in\left[0, \frac{1}{2}\right], \\ \left\{\frac{x}{2}\right\}, & \text { otherwise, }\end{cases}
$$

and

$$
T x= \begin{cases}{\left[0, \frac{x}{3}\right],} & \text { if } x \in\left[0, \frac{1}{2}\right], \\ \left\{\frac{x}{4}\right\}, & \text { otherwise. }\end{cases}
$$

Define a binary relation $\mathcal{R}=\left\{(x, y) \in\left[0, \frac{1}{2}\right]^{2}: y \leqslant x\right\}$. If, $x_{0}=\frac{1}{4}, x_{1}=\frac{1}{12}$, and $r=\frac{1}{4}$, then $B_{\mathrm{qb}}\left[x_{0}, r\right]=\left[0, \frac{1}{2}\right]$. Note that, for any $(x, y) \in \mathcal{R}$, we have

and

$$
H_{q b}(S x, T y)=\left|\frac{x}{3}-\frac{y}{3}\right|^{2}=\frac{1}{9} d_{q b}(x, y) \leqslant \lambda d_{q b}(x, y)+\beta\left[d_{q b}(x, S x)+d_{q b}(y, T y)\right],
$$

$$
H_{q b}(T x, S y)=\left|\frac{x}{3}-\frac{y}{3}\right|^{2}=\frac{1}{9} d_{q b}(x, y) \leqslant \lambda d_{q b}(x, y)+\beta\left[d_{q b}(x, T x)+d_{q b}(y, S y)\right] .
$$

Thus $S$ and $T$ satisfy inequalities (3.1) and (3.2) for $\lambda=\frac{1}{9}$ and $\beta=\frac{1}{6}$ for any $x, y \in B_{q b}\left[x_{0}, r\right]$. Also, $S$ and $T$ are $\mathcal{R}$-dominated mappings on $B_{q b}\left[x_{0}, r\right]$. For $k=\frac{\lambda+\beta}{1-\beta}=\frac{1}{3}$, we have, $d_{q b}\left(x_{0}, x_{1}\right) \leqslant k(1-s k) r$. The mappings $S$ and $T$ are continuous. Indeed, let $\left\{x_{n}\right\}$ be a sequence in $B_{q b}\left[x_{0}, r\right]$ such that $x_{n} \rightarrow$ $x \in B_{\mathrm{qb}}\left[x_{0}, r\right]$. Then, $\mathrm{H}_{\mathrm{qb}}\left(S x_{n}, S x\right)=\mathrm{H}_{\mathrm{qb}}\left(\left\{0, \frac{x_{n}}{3}\right\},\left\{0, \frac{x}{3}\right\}\right)=\left|\frac{x_{n}}{3}-\frac{x}{3}\right|^{2} \rightarrow 0$ as $\mathrm{n} \rightarrow \infty$. Similarly, we have $\mathrm{H}_{\mathrm{qb}}\left(T x_{n}, T x\right) \rightarrow 0$ as $\mathrm{n} \rightarrow \infty$. Thus, all the conditions of Theorem 3.6 are satisfied on $\mathrm{B}_{\mathrm{qb}}\left[\mathrm{x}_{0}, \mathrm{r}\right]$. Moreover, $x^{*}=0$ is a common fixed point of $S$ and $T$ and $d(0,0)=0$.

In Example 3.8, the relation $\mathcal{R}$ is a partial order but Theorem 3.6 holds for any relation $\mathcal{R}$.

Definition 3.9. Let $X$ be a nonempty set. Then $\left(X, d_{q b}, \preceq\right)$ is called a partially ordered dq $b$-metric space if $(X, d)$ is a dq $b$-metric space and $(X, \preceq)$ a partially ordered space.

Definition 3.10. Let $\left(X, d_{q b}, \preceq\right)$ be a partially ordered dq b-metric space. We say that $T: X \rightarrow C B(X)$ is $\preceq$-dominated mapping if for each $x \in X$, we have $x \preceq u$ for any $u \in T x$.

Definition 3.11. Let $\left(X, d_{q b}, \preceq\right)$ be a partially ordered dq b-metric space. A sequence $\left\{x_{n}\right\} \subseteq X$ is called $\preceq$-preserving if $x_{n} \preceq x_{n+1}$ for all $n \in \mathbb{N}_{0}$. 
Corollary 3.12. Let $\left(\mathrm{X}, \mathrm{d}_{\mathrm{qb}}, \preceq\right)$ be a partially ordered left $\mathrm{K}$-sequentially complete $d q$ b-metric space with $\mathrm{x}_{0} \in \mathrm{X}$, and $\mathrm{S}$ and $\mathrm{T}$ be $\preceq$-dominated mappings on $\mathrm{B}_{\mathrm{qb}}\left[\mathrm{x}_{0}, \mathrm{r}\right]$. Suppose that there exist some constants $\lambda, \beta$ satisfying $0 \leqslant \lambda+2 \beta<1$ such that for any $(x, y) \in \mathrm{B}_{\mathrm{qb}}\left[\mathrm{x}_{0}, \mathrm{r}\right] \times \mathrm{B}_{\mathrm{qb}}\left[\mathrm{x}_{0}, \mathrm{r}\right] \cap \nabla \preceq$, the following conditions hold:

$$
H_{q b}(S x, T y) \leqslant \lambda d_{q b}(x, y)+\beta\left[d_{q b}(x, S x)+d_{q b}(y, T y)\right]
$$

and

$$
H_{q b}(T x, S y) \leqslant \lambda d_{q b}(x, y)+\beta\left[d_{q b}(x, T x)+d_{q b}(y, S y)\right]
$$

If there exists $\mathrm{x}_{1} \in \mathrm{S} \mathrm{x}_{0}$ such that

$$
d_{q b}\left(x_{0}, x_{1}\right) \leqslant k(1-s k) r,
$$

where $\mathrm{k}=\frac{\lambda+\beta}{1-\beta}$ and $\mathrm{s} \mathrm{k}<1$, then $\mathrm{S}$ and $\mathrm{T}$ have a common fixed point $\mathrm{x}^{*} \in \mathrm{B}_{\mathrm{qb}}\left[\mathrm{x}_{0}, \mathrm{r}\right]$ provided that either $\mathrm{S}$ and T are continuous or for any sequence $\left\{x_{n}\right\}$ in $B_{q b}\left[x_{0}, r\right]$ such that $\left\{x_{n}\right\}$ is $\preceq$-preserving and $x_{n} \rightarrow x \in B_{q b}\left[x_{0}, r\right]$, we have $x_{n} \preceq x$ for all $\mathrm{n} \in \mathbb{N}_{0}$. Moreover, if $\mathrm{S}$ and $\mathrm{T}$ are compact valued, then $\mathrm{d}_{\mathrm{qb}}\left(\mathrm{x}^{*}, \mathrm{x}^{*}\right)=0$.

Example 3.13. Let $X=\mathbb{R}^{+}$and $d_{q b}: X \times X \rightarrow \mathbb{R}^{+}$be defined by $d_{q b}(x, y)=|x-y|^{2}+\frac{y^{2}}{16}$. As $d_{q b}(2,0) \not \leq$ $d_{q b}(2,1)+d_{q b}(1,0)$, so $\left(X, d_{q b}\right)$ is not a metric space. Define the mappings $S, T: X \rightarrow C B(X)$ by

$$
S x= \begin{cases}{\left[0, \frac{x}{2}\right],} & \text { if } x \in\left[0, \frac{2}{3}\right], \\ {\left[\frac{2 x}{3}, \frac{4 x}{5}\right],} & \text { otherwise }\end{cases}
$$

and

$$
T x= \begin{cases}{\left[0, \frac{x}{4}\right],} & \text { if } x \in\left[0, \frac{2}{3}\right], \\ \left\{\frac{x}{8}\right\}, & \text { otherwise. }\end{cases}
$$

Define a relation $\preceq$ as $\preceq=\left\{(x, y) \in\left[0, \frac{2}{3}\right]^{2}: y \leqslant x\right\}$. Note that $\left(X, d_{q b}, \preceq\right)$ is a partially ordered left $K$ sequentially complete $d q b$-metric with $s=2$. If, $x_{0}=\frac{1}{3}, x_{1}=\frac{1}{6}$, and $r=\frac{1}{3}$, then $B_{q b}\left[x_{0}, r\right]=\left[0, \frac{2}{3}\right]$. Note that, for any $x \preceq y$, we have

$$
\begin{aligned}
\mathrm{H}_{\mathrm{qb}}(S x, T y)=\left|\frac{x}{2}-\frac{y}{4}\right|^{2}+\frac{y^{2}}{256} & \leqslant \frac{1}{4} d_{q b}(x, y)+\frac{1}{16}\left[d_{q b}(x, S x)+d_{q b}(y, T y)\right] \\
& =\lambda d_{q b}(x, y)+\beta\left[d_{q b}(x, S x)+d_{q b}(y, T y)\right]
\end{aligned}
$$

and

$$
\begin{aligned}
\mathrm{H}_{\mathrm{qb}}(T x, S y)=\left|\frac{x}{4}-\frac{y}{2}\right|^{2}+\frac{y^{2}}{64} & \leqslant \frac{1}{4} d_{q b}(x, y)+\frac{1}{16}\left[d_{q b}(x, T x)+d_{q b}(y, S y)\right] \\
& =\lambda d_{q b}(x, y)+\beta\left[d_{q b}(x, T x)+d_{q b}(y, S y)\right]
\end{aligned}
$$

Thus $S$ and $T$ satisfy inequalities (3.3) and (3.4) on $B_{q b}\left[x_{0}, r\right]$ for $\lambda=\frac{1}{4}$ and $\beta=\frac{1}{16}$. Also, $S$ and $T$ are $\preceq$ dominated mappings on $B_{q b}\left[x_{0}, r\right]$. For $k=\frac{\lambda+\beta}{1-\beta}=\frac{1}{3}$, we have $d_{q b}\left(x_{0}, x_{1}\right) \leqslant k(1-s k) r$. For any sequence $\left\{x_{n}\right\}$ in $B_{q b}\left[x_{0}, r\right]$ such that $\left\{x_{n}\right\}$ is $\preceq$-preserving and $x_{n} \rightarrow x \in B_{q b}\left[x_{0}, r\right]$, we have $x_{n} \preceq x$ for all $n \in \mathbb{N}_{0}$. Thus, all the conditions of corollary 3.12 are satisfied on $B_{q b}\left[x_{0}, r\right]$. Moreover, $x^{*}=0$ is a common fixed point of $S$ and $T$ and $d(0,0)=0$.

\section{Application to single-valued mappings}

In this section we obtain several common fixed point results of single-valued mappings in the setup of left K-sequentially complete dq b-metric spaces. These results extend, unify and generalize the results in [33, Theorem 2.2] and [4, Theorems 2.1 and 2.3], respectively.

Theorem 4.1. Let $\left(\mathrm{X}, \mathrm{d}_{\mathrm{qb}}\right)$ be a left $\mathrm{K}$-sequentially complete $d q$ b-metric space with $\mathrm{x}_{0} \in \mathrm{X}$ and $\alpha: \mathrm{X} \times \mathrm{X} \rightarrow[0, \infty)$. Suppose that $\mathrm{f}, \mathrm{g}: \mathrm{X} \rightarrow \mathrm{X}$ are $\alpha$-dominated mappings on $\mathrm{B}_{\mathrm{qb}}\left[\mathrm{x}_{0}, \mathrm{r}\right]$ such that for any $\mathrm{X}, \mathrm{y} \in \mathrm{B}_{\mathrm{qb}}\left[\mathrm{x}_{0}, \mathrm{r}\right]$ with $\alpha(x, y) \geqslant 1$, we have 


$$
d_{q b}(f x, g y) \leqslant \lambda d_{q b}(x, y)+\beta\left[d_{q b}(x, f x)+d_{q b}(y, g y)\right],
$$

and

$$
d_{q b}(g x, f y) \leqslant \lambda d_{q b}(x, y)+\beta\left[d_{q b}(x, g x)+d_{q b}(y, f y)\right],
$$

where $0 \leqslant \lambda+2 \beta<1$. If

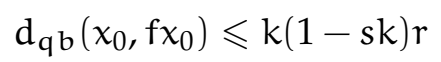

holds, where $\mathrm{k}=\frac{\lambda+\beta}{1-\beta}$, then there exists a point $x^{*} \in \mathrm{B}_{\mathrm{qb}}\left[\mathrm{x}_{0}, \mathrm{r}\right]$ such that $\mathrm{x}^{*}=\mathrm{fx^{* }}=\mathrm{gx} \mathrm{x}^{*}$ and $\mathrm{d}_{\mathrm{qb}}\left(\mathrm{x}^{*}, \mathrm{x}^{*}\right)=0$ provided that $f, g$ are continuous or for any sequence $\left\{x_{n}\right\}$ in $B_{q b}\left[x_{0}, r\right]$ with $\alpha\left(x_{n}, x_{n+1}\right) \geqslant 1$ for all $n \in \mathbb{N}_{0}$ and $x_{n} \rightarrow x \in B_{\mathrm{qb}}\left[x_{0}, r\right]$, we have $\alpha\left(x_{n}, x\right) \geqslant 1$ for all $n \in \mathbb{N}_{0}$.

Proof. Define $S, T: X \rightarrow C B(X)$ as $S x=\{f x\}$ and $T x=\{g x\}$. Note that $S$ and $T$ satisfy all the conditions of Theorem 2.1 and hence have a common fixed point $x^{*}$ in $B_{\mathrm{qb}}\left[x_{0}, r\right]$. Thus, $x^{*}=f x^{*}=g x^{*}$ and $d_{\mathrm{qb}}\left(x^{*}, x^{*}\right)=$ 0 .

Example 4.2. Let $X=Q^{+}$and $d_{q b}(x, y)=|x-y|^{3}+\frac{x}{32}$. Then $\left(X, d_{q b}\right)$ is a left $K$-sequentially complete dq $b$-metric space with $s=3$. Define the mappings $f, g: X \rightarrow X$ by

$$
f x=\left\{\begin{array}{ll}
\frac{x}{2}, & \text { if } x \in[0,1] \cap X, \\
2 x, & \text { if } x \in(1, \infty) \cap X,
\end{array} \quad \text { and } \quad g x= \begin{cases}\frac{x}{4}, & \text { if } x \in[0,1] \cap X, \\
3 x, & \text { if } x \in(1, \infty) \cap X .\end{cases}\right.
$$

Define $\alpha: X \times X \rightarrow \mathbb{R}^{+}$as:

$$
\alpha(x, y)= \begin{cases}2 x+y+1, & \text { if } x, y \in[0,1] \cap X \\ 0, & \text { if } x, y \in(1, \infty) \cap X\end{cases}
$$

If $x_{0}=\frac{1}{2}$ and $r=\frac{1}{2}$, then $B_{q b}\left[x_{0}, r\right]=[0,1] \cap X$. Note that $f, g$ are $\alpha$-dominated mappings on $B_{q b}\left[x_{0}, r\right]$. Also, $\lambda=\frac{1}{6}$ and $\beta=\frac{1}{15}$ give that $k=\frac{\lambda+\beta}{1-\beta}=\frac{1}{4}$ and

$$
\mathrm{d}_{\mathrm{qb}}\left(\mathrm{x}_{0}, \mathrm{fx}_{0}\right)=\mathrm{d}_{\mathrm{qb}}\left(\frac{1}{2}, \mathrm{f} \frac{1}{2}\right)=\mathrm{d}_{\mathrm{qb}}\left(\frac{1}{2}, \frac{1}{4}\right)=\frac{1}{32} \leqslant \frac{1}{4}\left(1-3\left(\frac{1}{4}\right)\right) \frac{1}{2}=\mathrm{k}(1-\mathrm{sk}) \mathrm{r} .
$$

When $x, y \in(1, \infty) \cap X$. Also,

$$
\begin{aligned}
d_{\mathrm{qb}}(f x, g y)=d_{\mathrm{qb}}(2 x, 3 y)=|2 x-3 y|^{3}+\frac{x}{16} & >\frac{1}{6} \mathrm{~d}_{\mathrm{qb}}(x, y)+\frac{1}{15}\left[\mathrm{~d}_{\mathrm{qb}}(x, f x)+\mathrm{d}_{\mathrm{qb}}(y, g y)\right] \\
& =\lambda \mathrm{d}_{\mathrm{qb}}(x, y)+\beta\left[\mathrm{d}_{\mathrm{qb}}(x, f x)+\mathrm{d}_{\mathrm{qb}}(y, g y)\right]
\end{aligned}
$$

and

$$
\begin{aligned}
d_{q b}(g x, f y)=d_{q b}(3 x, 2 y)=|3 x-2 y|^{3}+\frac{3 x}{32} & >\frac{1}{6} d_{q b}(x, y)+\frac{1}{15}\left[d_{q b}(x, g x)+d_{q b}(y, f y)\right] \\
& =\lambda d_{q b}(x, y)+\beta\left[d_{q b}(x, g x)+d_{q b}(y, f y)\right] .
\end{aligned}
$$

So the contractive conditions do not hold on $X-B_{q b}\left[x_{0}, r\right]$. However, for any $x, y \in B_{q b}\left[x_{0}\right.$, $\left.r\right]$, we have

$$
d_{q b}(f x, g y)=d_{q b}\left(\frac{x}{2}, \frac{y}{4}\right)=\left|\frac{x}{2}-\frac{y}{4}\right|^{3}+\frac{x}{64} \leqslant \lambda d_{q b}(x, y)+\beta\left[d_{q b}(x, f x)+d_{q b}(y, g y)\right]
$$

and

$$
d_{q b}(g x, f y)=d_{q b}\left(\frac{x}{4}, \frac{y}{2}\right)=\left|\frac{x}{4}-\frac{y}{2}\right|^{3}+\frac{x}{128} \leqslant \lambda d_{q b}(x, y)+\beta\left[d_{q b}(x, g x)+d_{q b}(y, f y)\right] .
$$

Thus, all the conditions of Theorem 4.1 are satisfied. Moreover, $x^{*}=0$ is a common fixed point of $f$ and $\mathrm{g}$ in $\mathrm{B}_{\mathrm{qb}}\left[\mathrm{x}_{0}, \mathrm{r}\right]$ and $\mathrm{d}_{\mathrm{qb}}(0,0)=0$. 
Corollary 4.3. Let $\left(\mathrm{X}, \mathrm{d}_{\mathrm{qb}}\right)$ be a left $\mathrm{K}$-sequentially complete $d q$ b-metric space and $\alpha: \mathrm{X} \times \mathrm{X} \rightarrow \mathbb{R}^{+}$. Suppose that $f, g: X \rightarrow X$ are $\alpha$-dominated mappings on $X$ such that for any $x, y \in X$ with $\alpha(x, y) \geqslant 1$, we have

$$
d_{q b}(f x, g y) \leqslant \lambda d_{q b}(x, y)+\beta\left[d_{q b}(x, f x)+d_{q b}(y, g y)\right],
$$

and

$$
d_{q b}(g x, f y) \leqslant \lambda d_{q b}(x, y)+\beta\left[d_{q b}(x, g x)+d_{q b}(y, f y)\right],
$$

where $0 \leqslant \lambda+2 \beta<1$. If, either $f, g$ are continuous or for any sequence $\left\{x_{n}\right\}$ in $X$ satisfying $\alpha\left(x_{n}, x_{n+1}\right) \geqslant 1$ for all $n \in \mathbb{N}_{0}$ and $x_{n} \rightarrow x \in X$ give that $\alpha\left(x_{n}, x\right) \geqslant 1$ for all $n \in \mathbb{N}_{0}$. Then $f$ and $g$ have a common fixed point $x^{*} \in \mathrm{X}$ and $\mathrm{d}_{\mathrm{qb}}\left(\mathrm{x}^{*}, \mathrm{x}^{*}\right)=0$.

Corollary 4.4. Let $\left(\mathrm{X}, \mathrm{d}_{\mathrm{qb}}\right)$ be a left $\mathrm{K}$-sequentially complete $d q$ b-metric space with $\mathrm{x}_{0} \in \mathrm{X}$ and $\alpha: \mathrm{X} \times \mathrm{X} \rightarrow$ $[0, \infty)$. Suppose that $\mathrm{f}: \mathrm{X} \rightarrow \mathrm{X}$ is $\alpha$-dominated mapping on $\mathrm{B}_{\mathrm{qb}}\left[\mathrm{x}_{0}, \mathrm{r}\right]$ such that for any $\mathrm{x}, \mathrm{y} \in \mathrm{B}_{\mathrm{qb}}\left[\mathrm{x}_{0}, \mathrm{r}\right]$ with $\alpha(x, y) \geqslant 1$, we have

$$
d_{q b}(f x, f y) \leqslant \lambda d_{q b}(x, y)+\beta\left[d_{q b}(x, f x)+d_{q b}(y, f y)\right],
$$

where $0 \leqslant \lambda+2 \beta<1$. If

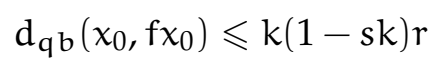

holds, where $\mathrm{k}=\frac{\lambda+\beta}{1-\beta}$, then $\mathrm{f}$ has a fixed point $\mathrm{x}^{*}$ in $\mathrm{B}_{\mathrm{qb}}\left[\mathrm{x}_{0}, \mathrm{r}\right]$ and $\mathrm{d}_{\mathrm{qb}}\left(\mathrm{x}^{*}, \mathrm{x}^{*}\right)=0$ provided that either $\mathrm{f}$ is continuous or for any sequence $\left\{x_{n}\right\}$ in $B_{\mathrm{qb}}\left[x_{0}, r\right]$ with $\alpha\left(x_{n}, x_{n+1}\right) \geqslant 1$ for all $n \in \mathbb{N}_{0}$ and $x_{n} \rightarrow x \in B_{q b}\left[x_{0}, r\right]$ imply that $\alpha\left(x_{n}, x\right) \geqslant 1$ for all $n \in \mathbb{N}_{0}$.

Corollary 4.5. Let $\left(\mathrm{X}, \mathrm{d}_{\mathrm{qb}}\right)$ be a left $\mathrm{K}$-sequentially complete $\mathrm{dq}$ b-metric space and $\alpha: \mathrm{X} \times \mathrm{X} \rightarrow \mathbb{R}^{+}$. Suppose that $\mathrm{f}: \mathrm{X} \rightarrow \mathrm{X}$ is $\alpha$-dominated mapping on $\mathrm{X}$ such that for any $\mathrm{x}, \mathrm{y} \in \mathrm{X}$ with $\alpha(\mathrm{x}, \mathrm{y}) \geqslant 1$, we have

$$
d_{q b}(f x, f y) \leqslant \lambda d_{q b}(x, y)+\beta\left[d_{q b}(x, f x)+d_{q b}(y, f y)\right],
$$

where $0 \leqslant \lambda+2 \beta<1$. If, either $f$ is continuous or for any sequence $\left\{x_{n}\right\}$ in $X$ with $\alpha\left(x_{n}, x_{n+1}\right) \geqslant 1$ for all $n \in \mathbb{N}_{0}$ and $\mathrm{x}_{\mathrm{n}} \rightarrow \mathrm{x} \in \mathrm{X}$ imply that $\alpha\left(\mathrm{x}_{\mathrm{n}}, \mathrm{x}\right) \geqslant 1$ for all $\mathrm{n} \in \mathbb{N}_{0}$, then $\mathrm{f}$ has a fixed point $\mathrm{x}^{*} \in \mathrm{X}$ and $\mathrm{d}_{\mathrm{qb}}\left(\mathrm{x}^{*}, \mathrm{x}^{*}\right)=0$.

Proof. Fix $x_{0} \in X$ and choose $r>0$ such that

$$
d_{q b}\left(x_{0}, f x_{0}\right) \leqslant k(1-s k) r
$$

where $k=\frac{\lambda+\beta}{1-\beta}$. The result follows from Corollary 4.4 .

Theorem 4.6. Let $\left(\mathrm{X}, \mathrm{d}_{\mathrm{q}}, \mathcal{R}\right)$ be a left $\mathrm{K}$-sequentially complete $d q$-metric space endowed with $\mathcal{R}$ with $\mathrm{x}_{0} \in \mathrm{X}$ and $\mathrm{f}, \mathrm{g}: \mathrm{X} \rightarrow \mathrm{X}$ be $\mathcal{R}$-dominated mappings on $\mathrm{B}_{\mathrm{qb}}\left[\mathrm{x}_{0}, \mathrm{r}\right]$. Suppose that there exist some constants $\lambda, \beta$ satisfying $0 \leqslant \lambda+2 \beta<1$ such that for any $(x, y) \in B_{\mathrm{qb}}\left[\mathrm{x}_{0}, \mathrm{r}\right] \times \mathrm{B}_{\mathrm{qb}}\left[\mathrm{x}_{0}, \mathrm{r}\right] \cap \nabla \mathcal{R}$, we have

$$
d_{q b}(f x, g y) \leqslant \lambda d_{q b}(x, y)+\beta\left[d_{q b}(x, f x)+d_{q b}(y, g y)\right],
$$

and

$$
d_{q b}(g x, f y) \leqslant \lambda d_{q b}(x, y)+\beta\left[d_{q b}(x, g x)+d_{q b}(y, f y)\right]
$$

If

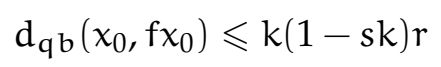

holds, where $\mathrm{k}=\frac{\lambda+\beta}{1-\beta}$ and $\mathrm{sk}<1$, then $\mathrm{f}$ and $\mathrm{g}$ have a common fixed point $\mathrm{x}^{*}$ in $\mathrm{B}_{\mathrm{qb}}\left[\mathrm{x}_{0}, \mathrm{r}\right]$ and $\mathrm{d}_{\mathrm{qb}}\left(\mathrm{x}^{*}, \mathrm{x}^{*}\right)=0$ provided that either $\mathrm{f}$ and $\mathrm{g}$ are continuous or for any sequence $\left\{\mathrm{x}_{\mathrm{n}}\right\}$ in $\mathrm{B}_{\mathrm{qb}}\left[\mathrm{x}_{0}, \mathrm{r}\right]$ such that $\left\{\mathrm{x}_{\mathrm{n}}\right\}$ is $\mathcal{R}$-preserving and $x_{\mathrm{n}} \rightarrow \mathrm{x} \in \mathrm{B}_{\mathrm{qb}}\left[\mathrm{x}_{0}, \mathrm{r}\right]$, we have $\left(\mathrm{x}_{\mathrm{n}}, \mathrm{x}\right) \in \mathcal{R}$ for all $\mathrm{n} \in \mathbb{N}_{0}$.

Proof. The result follows from Theorem 4.1. 
Corollary 4.7. Let $\left(\mathrm{X}, \mathrm{d}_{\mathrm{qb}}, \preceq\right)$ be a partially ordered left $\mathrm{K}$-sequentially complete $d q$ b-metric space with $\mathrm{x}_{0} \in \mathrm{X}$, and $\mathrm{f}$ and $\mathrm{g}$ be $\preceq$-dominated mappings on $\mathrm{B}_{\mathrm{qb}}\left[\mathrm{x}_{0}, \mathrm{r}\right]$. Suppose that there exist some constants $\lambda, \beta$ satisfying $0 \leqslant \lambda+2 \beta<1$ such that for any $(x, y) \in B_{\mathrm{qb}}\left[\mathrm{x}_{0}, \mathrm{r}\right] \times \mathrm{B}_{\mathrm{qb}}\left[\mathrm{x}_{0}, \mathrm{r}\right] \cap \nabla \preceq$, we have

$$
d_{q b}(f x, g y) \leqslant \lambda d_{q b}(x, y)+\beta\left[d_{q b}(x, f x)+d_{q b}(y, g y)\right],
$$

and

$$
d_{q b}(g x, f y) \leqslant \lambda d_{q b}(x, y)+\beta\left[d_{q b}(x, g x)+d_{q b}(y, f y)\right]
$$

If

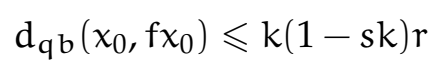

holds, where $\mathrm{k}=\frac{\lambda+\beta}{1-\beta}$ and $\mathrm{sk}<1$, then $\mathrm{f}$ and $\mathrm{g}$ have a common fixed point $\mathrm{x}^{*}$ in $\mathrm{B}_{\mathrm{qb}}\left[\mathrm{x}_{0}, \mathrm{r}\right]$ and $\mathrm{d}_{\mathrm{qb}}\left(\mathrm{x}^{*}, \mathrm{x}^{*}\right)=0$ provided that either $\mathrm{f}$ and $\mathrm{g}$ are continuous or for any sequence $\left\{\mathrm{x}_{\mathrm{n}}\right\}$ in $\mathrm{B}_{\mathrm{qb}}\left[\mathrm{x}_{0}, \mathrm{r}\right]$ such that $\left\{\mathrm{x}_{\mathrm{n}}\right\}$ is $\preceq$-preserving and $\mathrm{x}_{\mathrm{n}} \rightarrow \mathrm{x} \in \mathrm{B}_{\mathrm{qb}}\left[\mathrm{x}_{0}, \mathrm{r}\right]$, we have $\mathrm{x}_{\mathrm{n}} \preceq \mathrm{x}$ for all $\mathrm{n} \in \mathbb{N}_{0}$.

Proof. The result follows from Theorem 4.6.

Example 4.8. Let $X=\mathbb{R}^{+}$. Define $d_{q b}: X \times X \rightarrow \mathbb{R}^{+}$by $d_{q b}(x, y)=\left|\frac{x}{8}-y\right|^{2}$. Define the order $\preceq$ on $X$ as $x \preceq y$ if $d_{q b}(y, y) \leqslant d_{q b}(x, x)$ for all $x, y \in X$. Then $\left(X, d_{q b}, \preceq\right)$ is a partially ordered left K-sequentially complete dq $b$-metric space with $s=2$. Let $f, g: X \rightarrow X$ be defined by

$$
f x=\left\{\begin{array}{ll}
\frac{x}{4}, & \text { if } x \in[0,2], \\
x+\frac{1}{3}, & \text { if } x \in(2, \infty),
\end{array} \text { and } g x= \begin{cases}\frac{x}{8}, & \text { if } x \in[0,2] \\
x+\frac{1}{4}, & \text { if } x \in(2, \infty)\end{cases}\right.
$$

For $x_{0}=1, r=1, \lambda=\frac{1}{6}$, and $\beta=\frac{1}{8}$, we have $k=\frac{1}{3}, B_{q b}\left[x_{0}, r\right]=[0,2]$, and $d_{q b}\left(x_{0}, f x_{0}\right)=\frac{1}{64} \leqslant \frac{1}{9}=$ $k(1-s k) r$. Clearly, $f$ and $g$ are $\preceq$-dominated mappings and the contractive conditions hold on $B_{q b}\left[x_{0}, r\right]$. Therefore, all the conditions of Corollary 4.7 are satisfied. Moreover, 0 is the common fixed point of $f$ and $g$ and $d_{q b}(0,0)=0$.

Corollary 4.9. Let $\left(\mathrm{X}, \mathrm{d}_{\mathrm{q} \mathrm{b}}, \preceq\right)$ be a partially ordered left $\mathrm{K}$-sequentially complete $d q$ b-metric space, and $\mathrm{f}$ and $\mathrm{g}$ be $\preceq$-dominated mappings on $X$. Suppose that there exist some constants $\lambda, \beta$ satisfying $0 \leqslant \lambda+2 \beta<1$ such that for any $(x, y) \in \nabla \preceq$, we have

$$
d_{q b}(f x, g y) \leqslant \lambda d_{q b}(x, y)+\beta\left[d_{q b}(x, f x)+d_{q b}(y, g y)\right],
$$

and

$$
d_{q b}(g x, f y) \leqslant \lambda d_{q b}(x, y)+\beta\left[d_{q b}(x, g x)+d_{q b}(y, f y)\right] .
$$

Then $\mathrm{f}$ and $\mathrm{g}$ have a common fixed point $\mathrm{x}^{*}$ in $\mathrm{X}$ and $\mathrm{d}_{\mathrm{qb}}\left(\mathrm{x}^{*}, \mathrm{x}^{*}\right)=0$ provided that either $\mathrm{f}$ and $\mathrm{g}$ are continuous or for any sequence $\left\{x_{n}\right\}$ in $X$ such that $\left\{x_{n}\right\}$ is $\preceq$-preserving and $x_{n} \rightarrow x \in X$, we have $x_{n} \preceq x$ for all $n \in \mathbb{N}_{0}$.

If in Corollary 4.7 we choose $g=f$, then we obtain the following result.

Corollary 4.10. Let $\left(\mathrm{X}, \mathrm{d}_{\mathrm{qb}}, \preceq\right)$ be a partially ordered left $\mathrm{K}$-sequentially complete $d q$ b-metric space, and $\mathrm{f}$ be $\preceq$-dominated mapping on $\mathrm{B}_{\mathrm{qb}}\left[\mathrm{x}_{0}, \mathrm{r}\right]$. Suppose that there exist some constants $\lambda$, $\beta$ satisfying $0 \leqslant \lambda+2 \beta<1$ such that for any $(\mathrm{x}, \mathrm{y}) \in \mathrm{B}_{\mathrm{qb}}\left[\mathrm{x}_{0}, \mathrm{r}\right] \times \mathrm{B}_{\mathrm{qb}}\left[\mathrm{x}_{0}, \mathrm{r}\right] \cap \nabla \preceq$, we have

$$
d_{q b}(f x, f y) \leqslant \lambda d_{q b}(x, y)+\beta\left[d_{q b}(x, f x)+d_{q b}(y, f y)\right] .
$$

If

$$
\mathrm{d}_{\mathrm{qb}}\left(\mathrm{x}_{0}, f x_{0}\right) \leqslant k(1-\mathrm{sk}) \mathrm{r}
$$

holds, where $\mathrm{k}=\frac{\lambda+\beta}{1-\beta}$ and $\mathrm{sk}<1$, then $\mathrm{f}$ has a fixed point $\mathrm{x}^{*}$ in $\mathrm{B}_{\mathrm{qb}}\left[\mathrm{x}_{0}, \mathrm{r}\right]$ and $\mathrm{d}_{\mathrm{qb}}\left(\mathrm{x}^{*}, \mathrm{x}^{*}\right)=0$ provided that either $\mathrm{f}$ is continuous or for any sequence $\left\{x_{n}\right\}$ in $B_{q b}\left[x_{0}, r\right]$ such that $\left\{x_{n}\right\}$ is $\preceq$-preserving and $x_{n} \rightarrow x \in$ $\mathrm{B}_{\mathrm{qb}}\left[\mathrm{x}_{0}, \mathrm{r}\right]$, we have $\mathrm{x}_{\mathrm{n}} \preceq \mathrm{x}$ for all $\mathrm{n} \in \mathbb{N}_{0}$. 
If in Corollary 4.9 we choose $\mathrm{g}=\mathrm{f}$, then we obtain the following corollary which in turn generalizes results in $[24,25,27]$.

Corollary 4.11. Let $\left(\mathrm{X}, \mathrm{d}_{\mathrm{qb}}, \preceq\right)$ be a partially ordered left $\mathrm{K}$-sequentially complete $d q$ b-metric space, and $\mathrm{f}$ be $\preceq$-dominated mapping on $X$. Suppose that there exist some constants $\lambda$, $\beta$ satisfying $0 \leqslant \lambda+2 \beta<1$ such that for any $(x, y) \in \nabla \preceq$, we have

$$
d_{q b}(f x, f y) \leqslant \lambda d_{q b}(x, y)+\beta\left[d_{q b}(x, f x)+d_{q b}(y, f y)\right]
$$

Then $\mathrm{f}$ has a fixed point $\mathrm{x}^{*}$ in $\mathrm{X}$ and $\mathrm{d}_{\mathrm{qb}}\left(\mathrm{x}^{*}, \mathrm{x}^{*}\right)=0$ provided that either $\mathrm{f}$ is continuous or for any sequence $\left\{\mathrm{x}_{\mathrm{n}}\right\}$ in $X$ such that $\left\{x_{n}\right\}$ is $\preceq$-preserving and $x_{n} \rightarrow x \in X$, we have $x_{n} \preceq x$ for all $n \in \mathbb{N}_{0}$.

Proof. The result follows from Corollary 4.9.

\section{Acknowledgment}

The authors are sincerely grateful to the editor and referees for their careful reading, thoughtful suggestions and critical remarks for improving the quality of this manuscript. The third author is thankful to the financial assistance of the National Research Foundation (NRF) and DST-NRF Centre of Excellence in Mathematical and Statistical Sciences (CoE-MaSS).

\section{References}

[1] A. Alam, M. Imdad, Relation-theoretic contraction principle, J. Fixed Point Theory Appl., 17 (2015), 693-702. 3.2, 3.4

[2] M. A. Alghamdi, N. Hussain, P. Salimi, Fixed point and coupled fixed point theorems on b-metric-like spaces, J. Inequal. Appl., 2013 (2013), 25 pages. 1.2, 1

[3] T. V. An, L. Q. Tuyen, N. V. Dung, Stone-type theorem on b-metric spaces and applications, Topology Appl., 185/186 (2015), 50-64. 1, 1

[4] M. Arshad, A. Shoaib, I. Beg, Fixed point of a pair of contractive dominated mappings on a closed ball in an ordered dislocated metric space, Fixed Point Theory Appl., 2013 (2013), 15 pages. 1, 4

[5] A. Azam, M. Waseem, M. Rashid, Fixed point theorems for fuzzy contractive mappings in quasi-pseudo-metric spaces, Fixed Point Theory Appl., 2013 (2013), 14 pages. 1, 1

[6] I. A. Bakhtin, The contraction mapping principle in almost metric space, (Russian) Functional analysis, Ulyanovsk. Gos. Ped. Inst., Ulyanovsk, 30 (1989), 26-37. 1, 1.2, 1

[7] S. Banach, Sur les opérations dans les ensembles abstraits et leur application aux équations intégrales, Fund. Math., 3 (1922), 133-181. 1

[8] M. Boriceanu, Fixed point theory for multivalued generalized contraction on a set with two b-metrics, Stud. Univ. BabeşBolyai Math., 54 (2009), 3-14. 1

[9] L. B. Ćirić, Fixed points for generalized multi-valued contractions, Mat. Vesnik, 9 (1972) 265-272. 1

[10] S. Czerwik, Contraction mappings in b-metric spaces, Acta Math. Inform. Univ. Ostraviensis, 1 (1993), 5-11. 1, 1

[11] S. Czerwik, Nonlinear set-valued contraction mappings in b-metric spaces, Atti Sem. Mat. Fis. Univ. Modena, 46 (1998), 263-276. 1, 1

[12] P. Hitzler, Generalized metrics and topology in logic programming semantics, Ph.D. Thesis, School of Mathematics, Applied Mathematics and Statistics, National University Ireland, University College Cork, (2001). 1

[13] P. Hitzler, A. K. Seda, Dislocated topologies, J. Electr. Eng., 51 (2000), 3-7. 1

[14] N. Hussain, D. Dorić, Z. Kadelburg, S. Radenović, Suzuki-type fixed point results in metric type spaces, Fixed Point Theory Appl., 2012 (2012), 12 pages. 1

[15] N. Hussain, J. R. Roshan, V. Parvaneh, M. Abbas, Common fixed point results for weak contractive mappings in ordered b-dislocated metric spaces with applications, J. Inequal. Appl., 2013 (2013), 21 pages. 1

[16] N. Hussain, P Salimi, A. Latif, Fixed point results for single and set-valued $\alpha-\eta-\psi$-contractive mappings, Fixed Point Theory Appl., 2013 (2013), 23 pages. 1

[17] R. Kannan, Some results on fixed points, II, Amer. Math. Monthly, 76 (1969), 405-408. 1, 1

[18] C. Klin-eam, C. Suanoom, Dislocated quasi-b-metric spaces and fixed point theorems for cyclic contractions, Fixed Point Theory Appl., 2015 (2015), 12 pages. 1.2, 1.4, 1

[19] A. Latif, A. A. N. Abdou, Multivalued generalized nonlinear contractive maps and fixed points, Nonlinear Anal., 74 (2011), 1436-1444. 1

[20] A. Latif, D. T. Luc, Variational relation problems: existence of solutions and fixed points of contraction mappings, Fixed Point Theory Appl., 2013 (2013), 10 pages. 
[21] A. Latif, I. Tweddle, Some results on coincidence points, Bull. Austral. Math. Soc., 59 (1999), 111-117. 1

[22] S. Lipschutz, Schaum's outline of theory and problems of set theory and related topics, McGraw-Hill, New York, (1964). 3.1, 3.3

[23] S. B. Nadler, Jr., Multi-valued contraction mappings, Pacific J. Math., 30 (1969), 475-488. 1, 1, 1

[24] J. J. Nieto, R. Rodríguez-López, Contractive mapping theorems in partially ordered sets and applications to ordinary differential equations, Order, 22 (2005), 223-239. 4

[25] J. J. Nieto, R. Rodríguez-López, Existence and uniqueness of fixed point in partially ordered sets and applications to ordinary differential equations, Acta Math. Sin. (Engl. Ser.), 23 (2007), 2205-2212. 4

[26] M. U. Rahman, M. Sarwar, Dislocated quasi b-metric space and fixed point theorems, Electron. J. Math. Anal. Appl., 4 (2016), 16-24. 1.6

[27] A. C. M. Ran, M. C. B. Reurings, A fixed point theorem in partially ordered sets and some applications to matrix equations, Proc. Amer. Math. Soc., 132 (2003), 1435-1443. 4

[28] I. L. Reilly, P. V. Subrahmanyam, M. K. Vamanamurthy, Cauchy sequences in quasipseudometric spaces, Monatsh. Math., 93 (1982), 127-140. 1

[29] J. R. Roshan, N. Hussain, S. Sedghi, N. Shobkolaei, Suzuki-type fixed point results in b-metric spaces, Math. Sci. (Springer), 9 (2015), 153-160. 1

[30] J. R. Roshan, V. Parvaneh, I. Altun, Some coincidence point results in ordered b-metric spaces and applications in a system of integral equations, Appl. Math. Comput., 226 (2014), 725-737. 1

[31] B. Samet, M. Turinici, Fixed point theorems on a metric space endowed with an arbitrary binary relation and applications, Commun. Math. Anal., 13 (2012), 82-97. 3.1

[32] M. H. Shah, N. Hussain, Nonlinear contractions in partially ordered quasi b-metric spaces, Commun. Korean Math. Soc., 27 (2012), 117-128. 1.2, 1

[33] A. Shoaib, M. Arshadatjana, S. Radenović, $\alpha$-dominated mappings, dislocated metric spaces and fixed point results, Fixed Point Theory Appl., to be appeared. 1, 4

[34] W. A. Wilson, On quasi-metric spaces, Amer. J. Math., 53 (1931), 675-684. 1

[35] F. M. Zeyada, G. H. Hassan, M. A. Ahmed, A generalization of a fixed point theorem due to Hitzler and Seda in dislocated quasi-metric spaces, Arab. J. Sci. Eng. Sect. A Sci., 31 (2006), 111-114. 1.1, 1

[36] C.-X. Zhu, C.-F. Chen, X.-Z. Zhang, Some results in quasi-b-metric-like spaces, J. Inequal. Appl., 2014 (2014), 8 pages. $1.5,1$ 